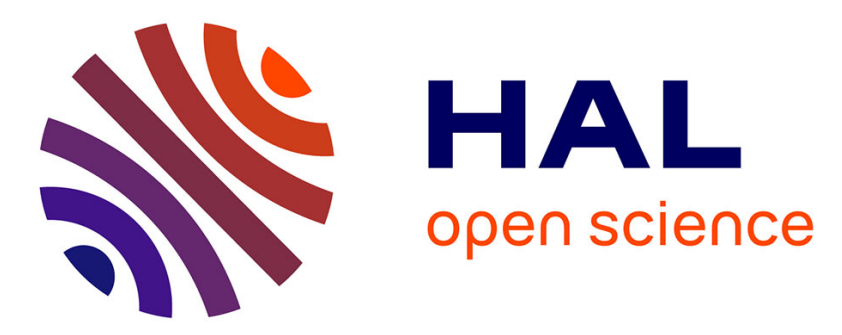

\title{
An artificial nonlinear diffusivity method for supersonic reacting flows with shocks
}

Benoit Fiorina, Sanjiva K. Lele

\section{To cite this version:}

Benoit Fiorina, Sanjiva K. Lele. An artificial nonlinear diffusivity method for supersonic reacting flows with shocks. Journal of Computational Physics, 2006, 222, pp.246-264. 10.1016/j.jcp.2006.07.020 . hal-00260147

\section{HAL Id: hal-00260147 \\ https://hal.science/hal-00260147}

Submitted on 18 Mar 2008

HAL is a multi-disciplinary open access archive for the deposit and dissemination of scientific research documents, whether they are published or not. The documents may come from teaching and research institutions in France or abroad, or from public or private research centers.
L'archive ouverte pluridisciplinaire HAL, est destinée au dépôt et à la diffusion de documents scientifiques de niveau recherche, publiés ou non, émanant des établissements d'enseignement et de recherche français ou étrangers, des laboratoires publics ou privés. 


\title{
An artificial nonlinear diffusivity method for supersonic reacting flows with shocks
}

\author{
B. Fiorina ${ }^{a}$ and S. K. Lele ${ }^{b}$ \\ ${ }^{a}$ Center for Turbulence Research, Stanford University, Stanford, CA 94305-4035, \\ $U S A$ \\ ${ }^{\mathrm{b}}$ Department of Aeronautics and Astronautics and Department of Mechanical \\ Engineering, Stanford University, Stanford, CA 94305-4035, USA
}

\begin{abstract}
A computational approach for modeling interactions between shocks waves, contact discontinuities and reactions zones with a high-order compact scheme is investigated. To prevent the formation of spurious oscillations around shocks, artificial nonlinear viscosity [1], based on high-order derivative of the strain rate tensor is used. To capture temperature and species discontinuities a nonlinear diffusivity based on the entropy gradient is added. It is shown that the damping of "wiggles" is controlled by the model constants and is largely independent of the mesh size and the shock strength. The same holds for the numerical shock thickness and allows a determination of the L2 error. In the shock tube problem, with fluids of different initial entropy separated by the diaphragm, an artificial diffusivity is required to accurately capture the contact surface. Finally, the method is applied to a shock wave propagating into a medium with non-uniform density/entropy and to a CJ detonation wave. Multi-dimensional formulation of the model is presented and is illustrated by a $2 \mathrm{D}$ oblique wave reflection from an inviscid wall, by a $2 \mathrm{D}$ supersonic blunt body flow and by a Mach reflection problem .
\end{abstract}

Key words: high-order scheme, shock capturing, supersonic combustion, nonlinear diffusivity

PACS:

\section{Introduction}

Supersonic combustion involves complex interactions between turbulence, shock waves and combustion. Because of their capability to reproduce unsteady ef-

Email address: benoit.fiorina@em2c.ecp.fr (B. Fiorina). 
fects, Direct (DNS) or Large-Eddy Numerical Simulations (LES) are attractive to model such supersonic reactive flows. In order to capture the physically important turbulent and chemical scales, such simulations require the use of accurate numerical schemes. As they can reproduce a wide range of wavenumbers, compact schemes [2] are well adapted. Unfortunately, the use of high-order compact schemes to solve steep gradients like those in shock waves generates non-physical oscillations [3]. The objective of this article is to develop and to validate a numerical methodology adequate for resolving interactions between shocks, turbulence and combustion.

Several approaches that modify or adapt high-order schemes to capture shock waves have been proposed in the literature. For shock-turbulence interaction problems, Adams and Shariff [4,5] proposed a high-order compact-ENO scheme and later Pirozzoli [6] introduced a conservative compact-WENO scheme. Deng and Zhang [7] developed high-order compact schemes based on the weighted technique. Rizzetta et al. [8], proposed a hybrid compact-Roe approach in order to simulate a supersonic compression-ramp flow. Visbal and Gaitonde [9] introduced an adaptive filter methodology to maintain the same high-order compact scheme in all of the numerical domain. An attractive alternative to these has been proposed by Cook and Cabot $[1,10]$ who avoid the use of a shock detector by adding an artificial dissipation term. A nonlinear artificial viscosity, based on high-order derivatives of the strain rate tensor, is introduced. The capability of this approach to accurately treats shock-turbulence interaction was successfully demonstrated.

In the context of supersonic combustion, high temperature and species gradients are expected in addition to shock waves. In order to accurately predict the interactions between these phenomenon, the original methodology proposed by Cook and Cabot $[1,10]$ is extended. In addition to the nonlinear viscosity, an artificial diffusivity based on high-order derivatives of the entropy is introduced. Detailed analysis of the errors associated with shock-capturing and contact-surface capturing were conducted for the new scheme. These have shown that new scheme is able to capture both weak and strong shocks without any degradation of performance. Both the numerical shock thickness, which is related to the number of points used to 'capture' the discontinuity, and the magnitude of the spurious wiggles are shown to be largely independent of the mesh size and the shock/contact surface strength. The original methodology and its extension are tested on the shock tube problem. It is observed that when the temperature from each side of the shock is initially different the original method by Cook and Cabot $[1,10]$ is not sufficient. Introduction of an artificial diffusivity is required to accurately capture the contact surface. The Shu-Osher problem [11] which consists of a shock wave crossing sinusoidal density waves is then considered. This test case has been used by many authors $[4,10,11]$ for one dimensional shock turbulence interactions. The computation of a Chapman-Jouguet detonation wave demonstrates the capability 
of the model to reproduce interactions between shock wave and combustion. The formulation of the model in multiple dimensions is then proposed and is illustrated by the computations of a $2 \mathrm{D}$ oblique shock, a $2 \mathrm{D}$ blunt body flow and a Mach reflection problem.

\section{Shock capturing model: 1D formulation}

\subsection{Governing equations}

The governing equations of a one-dimensional compressible reactive flow are:

$$
\begin{array}{r}
\frac{\partial \rho}{\partial t}+\frac{\partial \rho u}{\partial x}=0 \\
\frac{\partial \rho u}{\partial t}+\frac{\partial}{\partial x}(\rho u u+p)=\frac{\partial}{\partial x}\left(\mu_{l} \frac{\partial u}{\partial x}\right) \\
\frac{\partial \rho E}{\partial t}+\frac{\partial}{\partial x}[(\rho E+p) u]=\frac{\partial}{\partial x}\left(\mu_{l} u \frac{\partial u}{\partial x}\right)+\frac{\partial}{\partial x}\left(\lambda \frac{\partial T}{\partial x}\right) \\
\frac{\partial \rho Y_{k}}{\partial t}+\frac{\partial}{\partial x}\left(\rho u Y_{k}\right)=\frac{\partial}{\partial x}\left(\rho D_{k} \frac{\partial Y_{k}}{\partial x}\right)+\dot{\omega}_{k} \\
\rho E=\frac{\rho R T}{\gamma-1}+\frac{1}{2} \rho u u+\rho \sum_{k=1}^{N_{s p}} Y_{k} h^{0}{ }_{k}
\end{array}
$$

Where $\rho$ is the density, $p$ is the pressure, $E$ is the total energy (per unit mass), $\gamma$ is the ratio of specific heats, $R$ is the gas constant, $T$ is the gas temperature, $\lambda$ is the thermal conductivity, $\mu_{l}$ is the fluid viscosity, $N_{s p}$ is the number of species, $Y_{k}$ is the mass fraction of the $k^{t h}$ species, $h^{0}{ }_{k}$ is the enthalpy of formation of $k^{t h}$ species, $D_{k}$ is the diffusivity of $k^{t h}$ species and $\dot{\omega}_{k}$ is its reaction rate. Ideal gas law has been assumed here for simplicity.

\subsection{Nonlinear artificial viscosity}

When numerical discontinuities such as those due to shocks waves are present, the application of a high-order compact scheme to solve the previous set of equations results in spurious oscillations. These oscillations can be damped by adding a artificial viscous term to the momentum and energy equations $[1,10]$. A grid-dependent artificial viscosity $\mu$, defined by the following relation, is 
introduced:

$$
\mu=C_{\mu} \rho(\Delta x)^{r+1} \overline{\left|\frac{\partial^{r} u}{\partial x^{r}}\right|}
$$

where $\Delta x$ is the grid spacing and $|f|$ is the absolute value of $f$. The overbar $\bar{f}$ denotes a truncated-Gaussian filter defined in [1]. $C_{\mu}$ is a model constant and $r$ is a user-specified integer.

For practical meshes, the discrete representation of shock waves involves numerical discontinuities in the velocity and pressure fields. In terms of Fourier analysis, these numerical discontinuities correspond to the largest wavenumbers. If $r$ is sufficiently high, $\mu$ will therefore be important in the location near the shock waves and close to zero in the rest of the flow.

\subsection{Nonlinear artificial diffusivity}

In practical non-isothermal configurations, high temperature gradients can exist without being associated to sharp velocity gradients. For instance, in contact surface regions, a temperature discontinuity exists, whereas both velocity and pressure fields vary smoothly. Since $\mu$ is only based on the velocity gradient, such discontinuities are not detected by Eq. (6). Therefore, a griddependent artificial diffusivity, based on the entropy gradient needs to be defined:

$$
\chi_{\rho}=C_{\rho} \frac{a_{0}}{c_{p}}(\Delta x)^{r+1} \overline{\left|\frac{\partial^{r} s}{\partial x^{r}}\right|}
$$

where $a_{0}$ is a reference speed of sound, $c_{p}$ is the specific heat at constant pressure, $s$ is the entropy (per unit mass), and $C_{\rho}$ is a model constant. In supersonic reactive flows, steep gradients in species mass fractions are also associated with entropy gradients. Therefore, a similar formulation can be employed to detect species discontinuities:

$$
\chi_{Y}=C_{Y} \frac{a_{0}}{c_{p}}(\Delta x)^{r+1} \overline{\left|\frac{\partial^{r} s}{\partial x^{r}}\right|}
$$

where only the model constant $C_{Y}$ differs from that in Eq. (7).

\subsection{Model implementation}

In the orginal model proposed by Cook and Cabot [10], artificial dissipative terms are added to the momentum and the energy equations. In order to activate the nonlinear diffusivity defined by Eq. (7) and (8), an artificial dissipation term is also added to the mass and species transport equations. The governing equations therefore become: 


$$
\begin{aligned}
\frac{\partial \rho}{\partial t}+\frac{\partial \rho u}{\partial x} & =\frac{\partial}{\partial x}\left(\chi_{\rho} \frac{\partial \rho}{\partial x}\right) \\
\frac{\partial \rho u}{\partial t}+\frac{\partial}{\partial x}(\rho u u+p) & =\frac{\partial}{\partial x}\left(\left(\mu+\mu_{l}\right) \frac{\partial u}{\partial x}\right) \\
\frac{\partial \rho E}{\partial t}+\frac{\partial}{\partial x}[(\rho E+p) u] & =\frac{\partial}{\partial x}\left(\left(\mu+\mu_{l}\right) u \frac{\partial u}{\partial x}\right) \\
+\frac{\partial}{\partial x}\left(\lambda \frac{\partial T}{\partial x}-\rho \sum_{k=1}^{N_{s p}} D_{k} h_{k} \frac{\partial Y_{k}}{\partial x}\right) & \\
\frac{\partial \rho Y_{k}}{\partial t}+\frac{\partial}{\partial x}\left(\rho u Y_{k}\right) & =\frac{\partial}{\partial x}\left(\left(\rho \chi_{Y}+\rho D_{k}\right) \frac{\partial Y_{k}}{\partial x}\right)+\dot{\omega}_{k} \\
\rho E & =\frac{\rho R T}{\gamma-1}+\frac{1}{2} \rho u u+\rho \sum Y_{k} h_{k}^{0}
\end{aligned}
$$

In order to demonstrate that the model is suitable for a large variety of flow conditions, $\mu_{l}, \lambda$ and $D_{k}$ are set to zero in the following test cases.

The addition of non physical mass fluxes in the mass balance Eq. 9 requires a further comment. Such a term can potentially cause a pressure imbalance and create additional consistency errors. However, as the artificial dissipation operator is scaled by $\Delta_{x}{ }^{(r+1)}$, the detrimental effects of the non-linear diffusivity are minimized when an appropriate large value of $r$ is chosen. Typically a value of $r \geq 4$ is used. To see the effect of the artificial diffusivity, we consider a ideal fluid moving at an uniform speed, at constant pressure and with a sinusoidal density field $\rho(x, 0)=\rho_{0}(1+0.2 \sin (k x))$ where $k=2 \pi$ and $0 \leq x \leq 5$. Three flow speed conditions are investigated that correspond to a Mach number of $0.1,1$ and 10. Spatial derivatives are computed with a 6 th order compact scheme. RMS error of density and velocity are plotted on figures 1 and 2, respectively for three different grids conditions corresponding to $\Delta_{x}=0.1,0.05$ and 0.025 . Solid and dashed lines are the solutions of equations 9-12 with model parameter $\left(C_{\mu}=0.0, C_{\rho}=0.0 ; r=5\right)$ and $\left(C_{\mu}=0.0, C_{\rho}=0.01 ; r=5\right)$, respectively. As expected, the non-linear diffusivity generates additional numerical error but its convergence correspond to $(\mathrm{r}+1)$, which is the same than the truncation error of the spatial scheme. For detailed chemistry computations where $N$ is the number of species, $N-1$ species transport equations are solved in addition to the continuity equation and the non-physical mass flux is associated with the $\mathrm{N}^{\text {th }}$ species. By choosing the $\mathrm{N}^{\text {th }}$ component to be a major non-reactive species $\left(\mathrm{N}_{2}\right.$ for instance in the case of air/fuel combustion), the additional numerical error induced by the non-linear diffusivity which is of the order of the truncation error of the spatial scheme, will not create any significant numerical artifacts.

For one-dimensional test cases, in order to have the same conditions as in the original studies of Cook and Cabot in [1] and [10], the spatial derivatives are 
computed with the 10th order compact scheme. A fourth order Runge-Kutta method is used for time advancement. Numerical stability is guaranteed by applying an eight-order compact filter to the conserved variables after each Runge-Kutta step [10]. It is important to note that high-order compact filter if applied to discontinuous data may cause spurious oscillations. Nevertheless, as it will be further demonstrated, the non-linear viscosity and diffusivity smear the discontinuity over a small number of grid points such that both compact derivative and filtering operators do not generate further overshoots.

Cook and Cabot [1] have demonstrated that for smooth flows in one dimension, a higher order of accuracy can be obtained by using an appropriate large value of $r$. In terms of practical use, setting $r$ to 5 allows accurate detection of the discontinuity without affecting the rest of the flow. The effects of the other model parameter $C_{\mu}$ (and $C_{\rho}$ and $C_{Y}$ for the extended model proposed here) were not investigated. Although one can anticipate that these coefficients determine the extent to which the discontinuity is smeared, it is less obvious how to estimate the overall error induced by the model. Before applying the model to complex test cases, an investigation of this point is proposed in the following section.

\section{Performance of the nonlinear viscosity/diffusivity model}

We first consider a stationary normal shock that corresponds to a pressure jump $p_{l} / p_{r}=4.3$, where subscripts $l$ and $r$ respectively denote the left and right shock conditions. The initial conditions were set equal to the RankineHugoniot solution. In this first computation, a constant mesh size $\Delta x=0.05$ is used. Since neither contact nor species discontinuity is present in this simple configuration, the nonlinear diffusivity is not required and is therefore turned off ( $C_{\rho}=C_{Y}=0$ ). This allows for a focus on the effect of nonlinear artificial viscosity. Fig. 3 shows the dimensionless pressure distribution in the physical space for different values of $C_{\mu}$ ranging between 0 and 2 . When the nonlinear viscosity model is turned off $\left(C_{\mu}=0\right)$, large amplitude wiggles surround the shock. As $C_{\mu}$ increases, the shock is smeared and the amplitude of these spurious oscillations decreases. A dimensionless maximum amplitude of the wiggles is defined by dividing the maximum wiggles amplitude by the pressure jump $\Delta p=p_{l}-p_{r}$. The variation of the normalized wiggles amplitude with $C_{\mu}$ is plotted in Fig. 4 for three mesh sizes $(\Delta x=0.025 ; 0.05 ; 0.1)$. Also shown is the data for a weaker shock condition $\left(p_{l} / p_{r}=1.4\right)$. It is observed that the damping of wiggles is primarily controlled by the model constant, $C_{\mu}$ and is largely independent of the mesh size and the shock strength. For $C_{\mu}=1$, the normalized wiggle amplitude is about $0.7 \%$ (this level of wiggles will be accepted as a practical compromise). In order to measure the impact of the artificial dissipation on the shock resolution, a dimensionless numerical shock 
thickness is introduced:

$$
\frac{\delta}{\Delta x}=\frac{\Delta p}{\left.\Delta x \frac{\partial p}{\partial x}\right|_{\max }}
$$

Figures 5 and 6 show the numerical shock thickness and the L2 error in pressure for the different shock and grid spacings in terms of $C_{\mu}$. It is observed that the thickness of the shock, which is related to the number of grid points used to capture the discontinuity, is controlled mainly by the dissipation strength. For a given $C_{\mu}$, the shock front is smeared approximately over the same number of grid points. For example, $99 \%$ of the profile is captured over 5 grid points when $C_{\mu}=1.0$ is used. The L2 error shows a similar dependence on $C_{\mu}$ as the numerical shock thickness. This relationship is explained later.

To test the nonlinear diffusivity model, a stationary surface discontinuity corresponding to a density jump $\rho_{l} / \rho_{r}=10$ is considered. A single-component fluid is used and $C_{Y}$ is set to 0 . The solution is computed for three different mesh sizes $(\Delta x=0.025 ; 0.05 ; 0.1)$ and for two density ratio conditions $\left(\rho_{l} / \rho_{r}=2 ; 10\right)$. The wiggle amplitude, the numerical front thickness and the L2 error for the density are plotted in Fig 7, 8 and 9, respectively. The results are qualitatively similar to the shock wave case. Both the wiggle amplitude and the numerical front thickness remain largely independent of the mesh size and the discontinuity strength.

If one assumes that the undesired wiggles are effectively damped by the artificial dissipation, the L2 error induced by the nonlinear viscosity/diffusivity model is primarily associated with the smearing of the discontinuity. Approximating the density field by the following relation:

$$
\rho_{a p p r}=\frac{\left(\rho_{l}+\rho_{r}\right)}{2}+\frac{\left(\rho_{l}-\rho_{r}\right)}{2} \operatorname{erf}\left(\frac{\sqrt{\pi} x}{\delta}\right)
$$

where $\delta$, the observed front thickness, allows an estimation of the L2 error. Fig. 10 compares the measured and the estimated L2 error for a range of $C_{\rho}$. It shows that Eq 15 gives a good approximation of the error induced by the model.

This analysis shows that for a given value of the model constants, the discontinuity is smeared over a fixed number of grid points and this smearing is the dominant L2 error. To demonstrate the capability of the present approach to solve various complex shocks configurations, the same constant values will be used in all simulation presented in this paper. As suggested in [10], $C_{\mu}$ is set to 1.00 with $r=5$. The constants $C_{\rho}$ and $C_{Y}$ are respectively set to 0.01 and 0.05 . As will be shown further, this choice of constant allows capturing of surface discontinuities (or steep entropy waves). The original nonlinear viscosity model proposed by Cook and Cabot [1] gives unsatisfactory spurious oscillations near the contact surfaces. 


\section{Results for more complex 1D shock wave problems}

In the following subsections, the accuracy of the present shock capturing scheme is demonstrated on more complex configurations. Flow and thermochemical variables are made dimensionless as follow [13]:

$$
\rho=\frac{\rho^{*}}{\rho_{r}}, \quad u=\frac{u *}{u_{r}}, \quad T=\frac{T *}{T_{r}}, \quad P=\frac{P^{*}}{\rho_{r} u_{r}{ }^{2}}, \quad E=\frac{E^{*}}{u_{r}{ }^{2}}
$$

where asterisk and subscript $\mathrm{r}$ respectively represent dimensional and freestream quantities. The equation of state is expressed in terms of the freestream Mach number $M_{r}$ :

$$
p=\frac{\rho T}{\gamma M_{r}^{2}}
$$

\subsection{Shock tube problem}

The first test case is a Mach 2 shock tube problem with an initial discontinuity at $x=0$. Left and right side initialization values are given in Table 0(a). Variables are made dimensionless according to Eq. (16). The same temperature is set on either side of the diaphragm, therefore the configuration gives rise to a weak contact discontinuity. The numerical method with nonlinear viscosity only is denoted NVI $\left(C_{\mu}=1.0, C_{\rho}=0\right)$ and the numerical method with both non linear viscosity and diffusivity is denoted NVIDI $\left(C_{\mu}=1.0, C_{\rho}=0.01\right)$. Simulations were carried out on an uniform mesh of 160 grid points and are analyzed at the time $\tau=1.8$. A comparison between the exact solution, the NVI and the NVIDI solutions for the pressure, the velocity, the density and the temperature is plotted in Fig. 11. A good agreement between the numerical simulation and the theory is observed, in particular both the positions of the contact surface and of the right propagating shock are well captured. The NVI and NVIDI methods give nearly identical solution except at the contact surface where small differences are observed. This is visible on the temperature plot of Fig. 11, where the NVIDI method improved slightly the contact discontinuity prediction. In this configuration, the pressure jump is challenging to capture but the contact discontinuity is weak, therefore it explains why the impact of the hyperdiffusivity is moderate.

A second shock tube problem with a stronger pressure ratio is computed. Initial value, specified in Table $0(\mathrm{~b})$, give rise to a stronger contact discontinuity which presents a density ratio of 2 . Simulations were carried out on an uniform mesh of 400 grid points and are analyzed at the time $\tau=4.5$. Due to the sharp pressure and density discontinuities at $(x=0, \tau=0)$, NVI alone has start up difficulty but NVIDI overcomes that problem. Comparisons be- 
tween theory and NVIDI solution are shown in Fig. 12. The numerical front thickness (in terms of grid spacing) of both the shock wave and the surface discontinuity are about 3, which is consistent with the analysis of section 3 . Maximal wiggles amplitude are observed for the density field in the vicinity of the surface discontinuity and are about $3 \%$.

In the third shock tube problem configuration, fluids of different initial entropy are now separated by the diaphragm. The (reference) free-stream Mach number is set to 1 . Initial values are specified in Table 0(c). Such changes in temperature are encountered inside a combustion chamber, where fresh gases and hot products are present. Simulations were performed on an uniform mesh of 150 grid points and are analyzed at the time $\tau=1.6$. Comparisons between theory, NVI and NVIDI methods are plotted in Fig. 13. The propagation of the shock wave is well captured by both models, but a large discrepancy is now observed in the contact surface region. Differences are most visible in the temperature plot, where the NVI method shows high amplitude spurious oscillation almost entirely damped by the NVIDI method.

\subsection{Shu-Osher problem}

The Shu-Osher problem [11], where a sinusoidal density field is crossed by a shock wave, is then investigated. In order to validate the shock-turbulence interactions, the original model presented by Cook and Cabot [10] was tested for this configuration. If the numerical dissipation is too high, the entropy waves will be damped. In this sense, this canonical test case allows an assessment of the effect of nonlinear viscosity/diffusivity on turbulence. The initial conditions are indicated on table 2. The free-stream Mach number is equal to 3. The simulation of this problem is performed on the same 200 grid points mesh which was used in [10]. Numerical simulation of this configuration on a 1600 grid points mesh by Adams and Stolz [14] with a fifth-order ENO-Roe scheme is chosen as a reference solution. The comparisons between the reference solution and the NVI and the NVIDI solution at $\tau=1.8$ is shown in Fig. 14. Predictions of the two models are very similar and in good agreement with the reference solution. Solutions obtained with this numerical approach shows that the addition of a non-linear diffusion term to the continuity equation prevents the formation of wiggles without any noticeable detrimental effect on the physical oscillations resulting from the interaction with the shock wave. We note in passing that if larger value of $C_{\rho}$ are used (e.g. $C_{\rho}=0.03$ ) the amplitude of the steep entropy waves shows noticeable departure from the reference solution. The choice of $C_{\rho}$ is thus a compromise between controlling density wiggles and capturing high wavenumber physical entropy/density waves. 


\subsection{Chapman-Jouguet detonation wave}

As a reactive test case, a Chapman Jouguet detonation wave is investigated. To recover the correct ZND structure, interactions between shock waves and combustion have to be accurately predicted [15]. We will assume for simplicity that the chemical kinetics are represented by a progress variable $c$ which is 0 in the fresh gases and 1 in the burnt products. The species transport equations (Eq. (12)) are then reduced to a single transport equation for the progress variable $c$ :

$$
\frac{\partial \rho c}{\partial t}+\frac{\partial}{\partial x}(\rho u c)=\frac{\partial}{\partial x}\left(\left(\chi_{c}\right) \frac{\partial Y_{c}}{\partial x}\right)+\dot{\omega}_{c}
$$

The reaction rate $\dot{\omega}_{c}$ is expressed by an Arrhenius law:

$$
\dot{\omega}_{c}=K_{0} \exp \left(-E^{+} / T\right)
$$

where $\mathrm{E}^{+}$is the activation energy and $\mathrm{K}_{0}$ the rate constant. After introducing the dimensionless heat release $q_{0}=q_{0}{ }^{*} / u_{r}{ }^{2}$, the equation of state become:

$$
\rho E=\frac{p}{\gamma-1}+\frac{1}{2} \rho u u+\rho q_{0} c
$$

The initial conditions consist of totally burnt gas on the left-hand side and unburnt gas on the right hand side. Values of density, velocity, pressure and progress variable are given in Table 3 . The other parameters are set to $E^{+}=$ $25, K_{0}=50000, q_{0}=25$ and the reference Mach number is 0.845 . These values are chosen so that the burnt and unburnt states are connected by a CJ detonation wave moving with a speed equal to 7.1247 . The simulation is performed on an uniform mesh of 800 grid points. Pressure, density, temperature and progress variable are plotted in Fig 15. The ZND structure is well predicted while the non linear viscosity/diffusivity model prevents the formation of spurious oscillations around the shock.

A highly resolved numerical solution on 1600 grid points is set as a reference solution. The L2 error of the density prediction relative to this solution can then be computed for the various mesh sizes. To separate the contribution of the chemistry resolution from that of the shock capturing approach, the domain is split into two regions from each side of the midpoint of the density discontinuity. The left part contains the chemical structure of the detonation, whereas the shock is included the right part. The total L2 error and its components are plotted in Fig. 16. Most of the contribution to the L2 error is due to the chemistry and not due to the shock. It means that the number of grid points required to solve this problem will not be restricted by the shock capturing but by the resolution of the chemical structure. For 
all mesh conditions the detonation speed is well captured. As it was demonstrated in section 3, the dimensionless shock thickness defined by Eq. (14) is quasi-constant $(\delta / D X \approx 2.5)$, meaning that the same number of grid points is used to capture the shock for the various grid sizes.

\section{Multi-dimensional formulation}

For multiple dimensions the flow equations including the artificial diffusivity and viscosity become:

$$
\begin{array}{r}
\frac{\partial \rho}{\partial t}+\nabla \cdot \rho u-\nabla\left(\chi_{\rho} \nabla \rho\right)=0 \\
\frac{\partial \rho u}{\partial t}+\nabla \cdot(\rho \cdot u u+p \underline{\delta}-\underline{\tau})=0 \\
\frac{\partial \rho E}{\partial t}+\nabla \cdot\left[\left(\rho E u+(p \underline{\delta}-\underline{\tau}) \cdot u-\lambda \nabla T+\rho \sum_{k=1}^{N_{s p}} D_{k} h_{k} \nabla Y_{k}\right]=0\right. \\
\frac{\partial \rho Y_{k}}{\partial t}+\nabla \cdot\left(\rho u Y_{k}\right)-\nabla\left(\left(\chi_{Y}+\rho D_{k}\right) \nabla Y_{k}\right)=\dot{\omega}_{k} \\
\rho E=\frac{\rho R T}{\gamma-1}+\frac{1}{2} \rho u u+\rho \sum Y_{k} h_{k}^{0}
\end{array}
$$

where $\underline{\delta}$ is the unit tensor and $\underline{\tau}$. According to [10], the non linear viscosity is split into a shear and a bulk viscosity respectively noted by $\mu_{s}$ and $\mu_{b}$. This technique allows to capture shocks without destroying vorticity. The viscous stress tensor $\mathcal{I}$ is then given by:

$$
\underline{\tau}=\left(\mu_{s}+\mu_{l}\right)(2 \underline{S})+\left(\mu_{b}-\frac{2}{3}\left(\mu_{s}+\mu_{l}\right)\right)(\nabla \cdot u) \underline{\delta}
$$

where $\underline{S}=0.5\left(\nabla u+(\nabla u)^{T}\right)$ is the strain rate tensor. Expression for $\mu_{s}$ and $\mu_{b}$ are given by:

$$
\mu_{s}=C_{\mu}^{s} \eta, \quad \mu_{b}=C_{\mu}^{b} \eta, \quad \eta=\rho \Delta^{r+1} \overline{\left|\nabla^{r-1} S\right|}
$$

where $C_{\mu}^{s}$ and $C_{\mu}^{b}$ are the model constants, $\Delta$ is the local grid spacing and $S=$ $(\underline{S}: \underline{S})^{1 / 2}$ is the magnitude of the strain rate tensor. $\nabla^{r-1}$ is the polyharmonic operator which denotes a sequence of Laplacians. For instance $r=5$ leads to $\nabla^{4} S=\nabla^{2}\left(\nabla^{2} S\right)$. The overbar $(\bar{f})$ denotes a truncated-Gaussian filter. The extension of the non linear diffusivity to multiple dimensions is done as follow:

$$
\chi_{\rho}=C_{\rho} \zeta, \quad \chi_{Y}=C_{Y} \zeta, \quad \zeta=\frac{a_{0}}{c_{p}}(\Delta)^{r+1} \overline{\left|\nabla^{r-1}\right| \nabla s||}
$$


where $C_{\rho}$ and $C_{Y}$ are the model constants, $|\nabla s|$ is the norm of the fluid entropy gradient, $a_{0}$ is the speed of sound and $c_{p}$ is the specific heat at constant pressure.

These numerical procedure has been implemented in the 3-D compressible Navier-Stokes solver FDL3DI [16] developed at the Air Force Research Laboratory. The spatial derivatives are computed with a 6 th order compact scheme [2] and a 8th order filtering is used for stability purpose [16]. The code is explicit in time using a fourth order Runge-Kutta method. Parameters used for the non linear viscosity model are $r=5, C_{\mu}^{s}=0.002, C_{\mu}^{b}=1$, as recommended in [10]. Concerning the artificial diffusivity components, we maintain the same parameters that was used in the $1 \mathrm{D}$ formulation, i.e. $r=5, C_{\rho}=0.01$ and $C_{Y}=0.05$.

\subsection{Oblique shock reflection}

The first 2D considered test case is the reflection of a shock wave on an inviscid wall. The shock angle is 33 degrees from the Mach 3 free-stream. The mesh size is $151 \times 51$ and is uniformly distributed in both directions. The jump conditions are imposed on the upper boundary whereas slip wall conditions are set at the bottom boundary. Pressure field is plotted in Fig 17 and 18. Although the shock wave is not aligned with the computational mesh, no significant wiggles are present around the discontinuity which is well captured. The results are in good agreement with the compact-Roe scheme solution presented in [9].

\subsection{Blunt-body flow}

As a second test case, a Mach 3 inviscid supersonic flow past a cylinder is computed. A 81 x 75 mesh, shown in Fig. 19 was generated analytically [17] for the upper half of the domain. Symmetric flow conditions are imposed at the centerline. The problem is initalized by a Mach 3 shock moving from the left while slip wall conditions are imposed at the surface of the cylinder. Isocontours of pressure are plotted in Fig. 19. Two radial profiles of pressure, corresponding to $\theta=0^{\circ}$ and $\theta=45^{\circ}$, where $\theta$ is defined in Fig. 19, are shown in Fig. 20. At the centerline, the shock, smeared over 4 grid points, is located at a distance of 1.7 from the cylinder, which is in good agreement whith the compact-Roe scheme results obtained by Visbal and Gaitonde [9] on the same configuration. The maximal wiggles amplitudes are maintained below $2 \%$. 


\subsection{Double Mach reflection}

The last test case has been initially used to compare several numerical scheme [18]. The computational domain has dimension $[0,4] \mathrm{X}[0,2]$. Grid spacing is $\Delta_{x}=\Delta_{y}=1 / 60$. The problem involves a Mach 10 shock wave in air $(\gamma=1.4)$ which initially makes a $60^{\circ}$ angle with the horizontal axis. The undisturbed air ahead of the shock has a density of 1.4 and a pressure of 1 . The shock intersects the axis at $x=1 / 6$. Along the bottom boundary, at $y=0$, the region from $x=0$ to $x=1 / 6$ is always assigned values for the initial postshock flow, whereas reflecting wall conditions are set from $x=1 / 6$ to $x=4$. The values along the top boundary condition are set to describe the exact motion of the Mach 10 shock. A steady 1D shock wave which corresponds to the normal jump conditions across the Mach 10 oblique shock was initially computed with the present shock capturing model. This 1D shock solution that was also been obtained with a grid spacing $\Delta_{x}=1 / 60$, has been used to properly interpolate the initial condition of the 2D Mach reflection problem.

Density contour of the solution at time $\tau=0.1$ and $\tau=0.2$, are respectively plotted on Fig. 21(a) and 21(b). Only the solution for $x \in[0,3]$ and $y \in[0,1]$ is shown. Visible on both figures is the formation of the two Mach stems and the wall jet. In particular, the propagation of the two Mach stems and the formation of the jet which are extremely difficult to compute are recovered. The results are very similar to solutions obtained on the same grid with a $5^{\text {th }}$ order WENO scheme [19] and a $7^{\text {th }}$ order TVD scheme [20]. A comparison with these results shows that the complex features of the flow are captured at the correct positions. The jet is better represented with the $6^{\text {th }}$ order shockcapturing scheme than with the 5th order WENO scheme [19], and the shocks patterns are slightly less smeared with the 7th order TVD scheme [20].

\section{Conclusions}

A new, simple nonlinear viscosity method has been developed for capturing shocks and contact surfaces in the context of supersonic reactive flows. By adding a nonlinear artificial diffusivity, it extends the nonlinear artificial viscosity method proposed by Cook and Cabot $[1,10]$ to treat entropy gradients associated with temperature and species discontinuities.

Detailed analysis of the errors associated with shock-capturing and contact-

surface capturing was conducted. These have shown that new scheme is able to capture both weak and strong shocks without any degradation of performance. Both the dimensionless numerical shock thickness, which is related to the number of points used to compute the discontinuity, and the damping of the 
spurious wiggles are shown to be largely independent of the mesh size and the shock/contact surface strength.

This model has been successfully applied to complex 1D shock wave problems, including the shock tube problem, the Shu Osher problem and a ChapmanJouguet detonation wave. It has been observed that the introduction of a nonlinear artificial diffusivity is required to accurately capture contact surface discontinuities. Multi-dimensional formulation of the model has been presented and was successfully applied to a 2D oblique shock wave, to a supersonic blunt body flow and to a Mach reflection problem. For pure gas-dynamic shock wave problems, other methodologies $[4,6,7,20]$ may be superior than the present approach. However, the method proposed in this paper is applicable to broader class of unsteady flow problems, such as those involving shocks, turbulence, combustion and their interaction, and is also considerably simpler to implement.

\section{Acknowledgment}

This work was supported by the AFOSR-MURI Grant. We are grateful to Dr. Miguel Visbal for providing the FDL3DI code which was modified in present work. We also acknowledge the efforts Dr. Daniel Bodony in porting this code. We are grateful to the referees for some helpful comments and specially for suggesting the test case discussed in Figs. 1 and 2.

\section{References}

[1] A. W. Cook, W. H. Cabot, A high-wavenumber viscosity for high resolution numerical method, J. Comput. Phys. 195 (2004) 594-601.

[2] S. K. Lele. Compact finite difference schemes with spectral-like resolution. J. Comput. Phys. 103 (1992) 16-42.

[3] T. Colonius and S. K. Lele. Computational aeroacoustics: progress on nonlinear problems of sound generation. Progress in Aerospace Sciences 40 (2004) 345416.

[4] N. A. Adams and K. Shariff. A High-Resolution Hybrid Compact-ENO Scheme for Shock Turbulence Interaction Problems. J. Comput. Phys., 127 (1996) 27-51.

[5] N. A. Adams. Direct numerical simulation of the turbulent boundary layer along a compression ramp at $\mathrm{M}=3$ and $\mathrm{Re}=1685$. J. Fluid Mech., 420 (2000) 47-83.

[6] S. J. Pirozzoli. Conservative Hybrid compact-WENO schemes for shockturbulence interaction. J. Comput. Phys., 178 (2002) 81-117. 
[7] X. G. Deng and H. X. Zhang. Developing high-order weighted compact nonlinear schemes. J. Comput. Phys., 165 (2000) 22-44.

[8] D. P. Rizzetta, M. R. Visbal and D. V. Gaitonde. Large-eddy simulation of supersonic compression ramp flow by high-order method. AIAA Journal, 39 (12):2283-2292, 2001.

[9] M. R. Visbal, D. V. Gaitonde. Shock capturing using compact-differentiatedbases method. , AIAA paper 2005-1265. 43rd AIAA Aerospace Sciences Meeting and Exhibit, 2005.

[10] A. W. Cook, W. H. Cabot, Hyperviscosity for shock-turbulence interactions, J. Comput. Phys. 203 (2005) 379-385.

[11] C. W. Shu, S.J. Osher, Efficient implementation of essentially nonoscillatory shock capturing schemes II, J. Comput. Phys. 83 (1989) 32-78.

[12] H. W. Liepman, A. Roshko, Elements of gas dynamics. John Willey \& Sons, Inc, 1957

[13] J. C. Tannehill, D. A. Anderson and R. H. Pletcher, Computational fluids mechanics and heat transfer. $2^{\text {nd }}$ ed., Taylor \& Francis, Washington DC, 1997.

[14] N. A. Adams, S. Stolz, A subgrid scale deconvolution for schock capturing. J. Comput. Phys. 178 (2002) 391-426.

[15] C. Helzel, R. J. Leveque and G. Warnecke. A modified fractional step method for the accurate approximation of detonation waves. SIAM J. Sci. Comput. Vol. 22 No 4 (2000) 1489-1510

[16] D. V. Gaitonde and M. R. Visbal. High-order schemes for NavierStokes equations: algorithm and implementation into FDL3DI. Technical Report AFRL-VA-WP-TR-1998-3060, Air Force Research Laboratory, WrightPatterson AFB, 1998.

[17] G. S. Jiang and C. W. Shu. Efficient implementation of weighted ENO scheme. J. Comput. Phys. 126 (1996) 202-228.

[18] P. Woodward and P. Collela. The numerical simulation of two-dimensional fluid flow with strong shock. J. Comput. Phys. 54 (1984) 115-173.

[19] A. Suresh and H. T. Huynh. Accurate monotonicity-preserving schemes with Runge-Kutta time stepping. J. Comput. Phys. 136 (1997) 83-99.

[20] V. Daru and C. Tenaud. High order one-step monotonicity-preserving schemes for unsteady compressible flow calculations. J. Comput. Phys. 193 (2004) 563594 . 


\section{List of Tables}

1 Left and right initial conditions for the shock tube problems 17

2 Left and right initial conditions for the Shu-Osher problem [11] 17

3 Left and right initial conditions for the CJ detonation wave 17 
(a) Case 1

\begin{tabular}{|c|c|c|}
\hline & left & right \\
\hline$p$ & 10 & 5 \\
\hline$\rho$ & 10 & 5 \\
\hline$u$ & 0 & 0 \\
\hline
\end{tabular}

(b) Case 2

\begin{tabular}{|c|c|c|}
\hline & left & right \\
\hline$p$ & 10 & 1 \\
\hline$\rho$ & 10 & 1 \\
\hline$u$ & 0 & 0 \\
\hline
\end{tabular}

(c) Case 3

\begin{tabular}{|c|c|c|}
\hline & left & right \\
\hline$p$ & 1.1 & 1 \\
\hline$\rho$ & 1.0 & 0.1 \\
\hline$u$ & 0 & 0 \\
\hline
\end{tabular}

Table 1

Left and right initial conditions for the shock tube problems

Table 2

\begin{tabular}{|c|c|c|}
\hline & left & right \\
\hline$p$ & 10.33333 & 1 \\
\hline$\rho$ & 3.857143 & $1+0.2 \sin (5 \mathrm{x})$ \\
\hline$u$ & 2.629369 & 0 \\
\hline
\end{tabular}

Left and right initial conditions for the Shu-Osher problem [11]

Table 3

\begin{tabular}{|c|c|c|}
\hline & left & right \\
\hline$p$ & 1 & 21.5672 \\
\hline$\rho$ & 1 & 1.6812 \\
\hline$u$ & -7.1247 & -4.238 \\
\hline$c$ & 1 & 0 \\
\hline
\end{tabular}

Left and right initial conditions for the CJ detonation wave 


\section{List of Figures}

1 RMS relative error in density for a convected sinusoidal density field. Solid lines are the solution without nonlinear diffusivity $\left(C_{\rho}=0.0\right)$. Dashed lines are the solution with nonlinear diffusivity $\left(C_{\rho}=0.01\right)$. Squares: Mach 0.1; Delta: Mach 1; Gradients: Mach 10.

2 RMS relative error in velocity for a convected sinusoidal density field computed with nonlinear diffusivity $\left(C_{\rho}=0.01\right)$. Squares: Mach 0.1; Delta: Mach 1; Gradients: Mac 10.

3 The effect of model constant $C_{\mu}$ on the pressure profile across a 1-D stationary normal shock wave. Dotted line: $C_{\mu}=0$; dashed line: $C_{\mu}=0.13$; dashed-dot line: $C_{\mu}=1.0$; solid line: $C_{\mu}=2.66$

4 Maximum normalized wiggle amplitude in $\%$ in terms of $C_{\mu}$ for the $1 \mathrm{D}$ stationary shock wave.

5 Numerical shock thickness in terms of $C_{\mu}$ for the 1D stationary shock wave.

6 L2 error in pressure in terms of $C_{\mu}$ for the 1D stationary shock wave.

7 Maximum normalized wiggle amplitude in $\%$ in terms of $C_{\rho}$ for the 1D stationary contact surface.

8 Numerical shock thickness in terms of $C_{\rho}$ for the 1D stationary contact surface.

9 L2 error in density in terms of $C_{\rho}$ for the 1D stationary contact surface.

10 L2 error in density in terms of $C_{\rho}$ for the 1D stationary contact surface. Solid line: Directly computed L2 error from the density field. Dashed lines: L2 error computed with the density estimated from Eq. (15); Squares: DX=0.05; Triangles: $\mathrm{DX}=0.1$ 
11 Numerical simulations of the shock tube problem with an initial pressure ratio of 2 . Pressure, density, velocity and temperature (zoomed in the contact discontinuity region) are expressed in terms of the physical space $X$. Thin solid line: theory; Thick dashed line: NVI model; Thick solid line: NVIDI model.

12 Numerical simulations of the shock tube problem with an initial pressure ratio of 10 . Pressure, density, velocity and temperature (zoomed in the contact discontinuity region) are expressed in terms of the physical space $X$. Thin solid line: theory; Thick solid line: NVIDI model.

13 Numerical simulations of the shock tube problem with a density factor of 10 . Pressure, density, velocity and temperature (zoomed in the contact discontinuity region) are expressed in terms of the physical space $X$. Thin solid line: theory; Thick dashed line: NVI model; Thick solid line: NVIDI model.

14 A normal Mach 3 shock with pressure ratio of 10.33 is propagating into a medium with non-uniform density/entropy. Solid and dashed lines are respectively the NVI and NVIDI density solutions obtained at a resolution of 200 grid points whereas symbols represent a reference solution [14] obtained on 1600 grid points with a fifth order ENO-Roe scheme.

15 Pressure, density, temperature, progress variable and reaction rate distribution for the Chapman-Jouguet detonation.

16 L2 error in density of the CJ case in terms of the mesh size. Line: total L2 error; Dashed line: contribution of the L2 error in the reaction zone; Dashed dotted line: Contribution of the L2 error in the shock region.

17 The interaction of an oblique shock with an inviscid wall. The shock angle is 33 degrees from the Mach 3 free-stream. Contours of normalized pressure are plotted.

18 Pressure distribution along $\mathrm{Y}=0.18$ line for Mach 3 inviscid shock reflection.

19 Mach 3 supersonic flow past a cylinder. Countours of normalized pressure are plotted. 
20 Radial profiles of pressure. Solid line: $\theta=0^{\circ}$; Dashed-dotted line: $\theta=45^{\circ}$.

21 Double Mach reflection. Density, 30 contours from 1.73 to 21. 


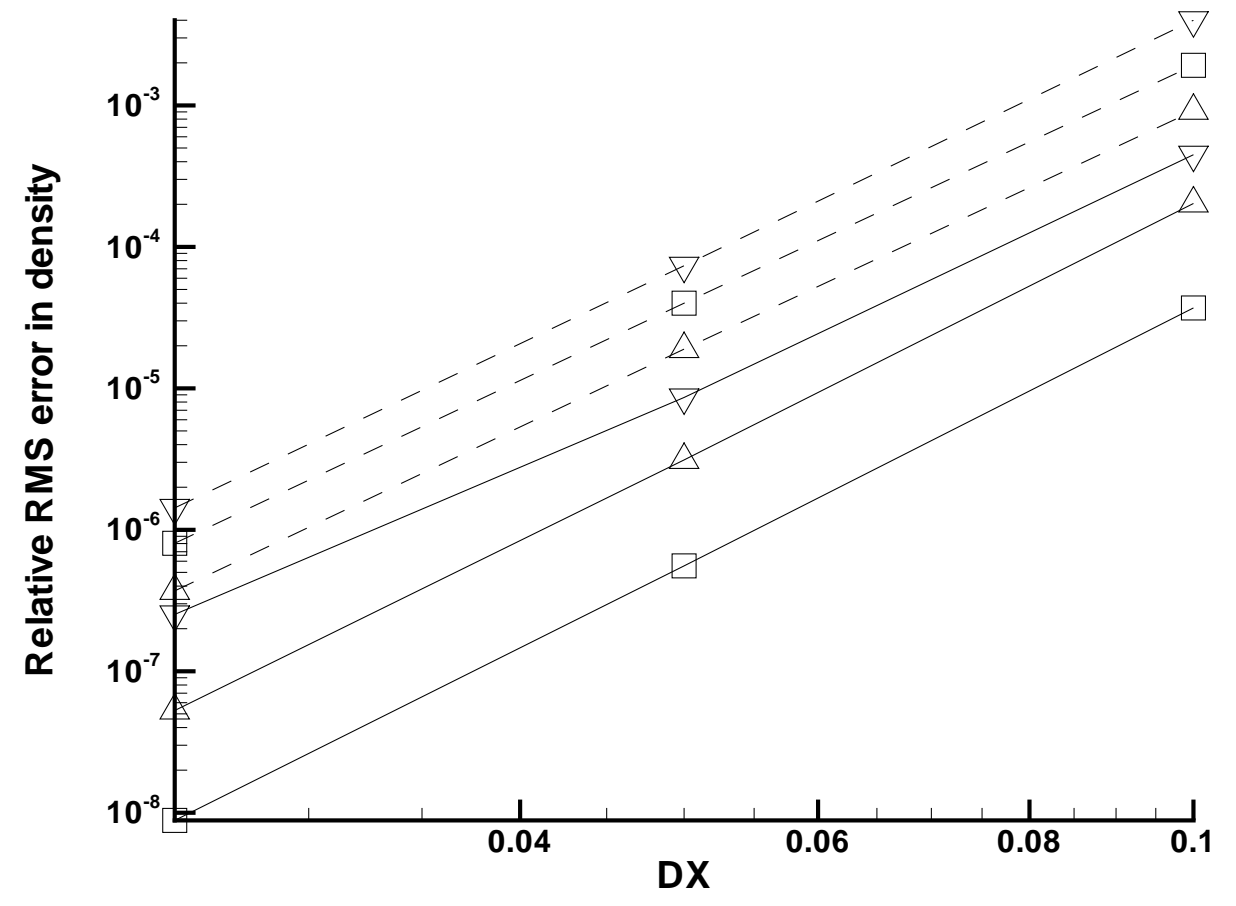

Fig. 1. RMS relative error in density for a convected sinusoidal density field. Solid lines are the solution without nonlinear diffusivity $\left(C_{\rho}=0.0\right)$. Dashed lines are the solution with nonlinear diffusivity $\left(C_{\rho}=0.01\right)$. Squares: Mach 0.1; Delta: Mach 1; Gradients: Mach 10. 


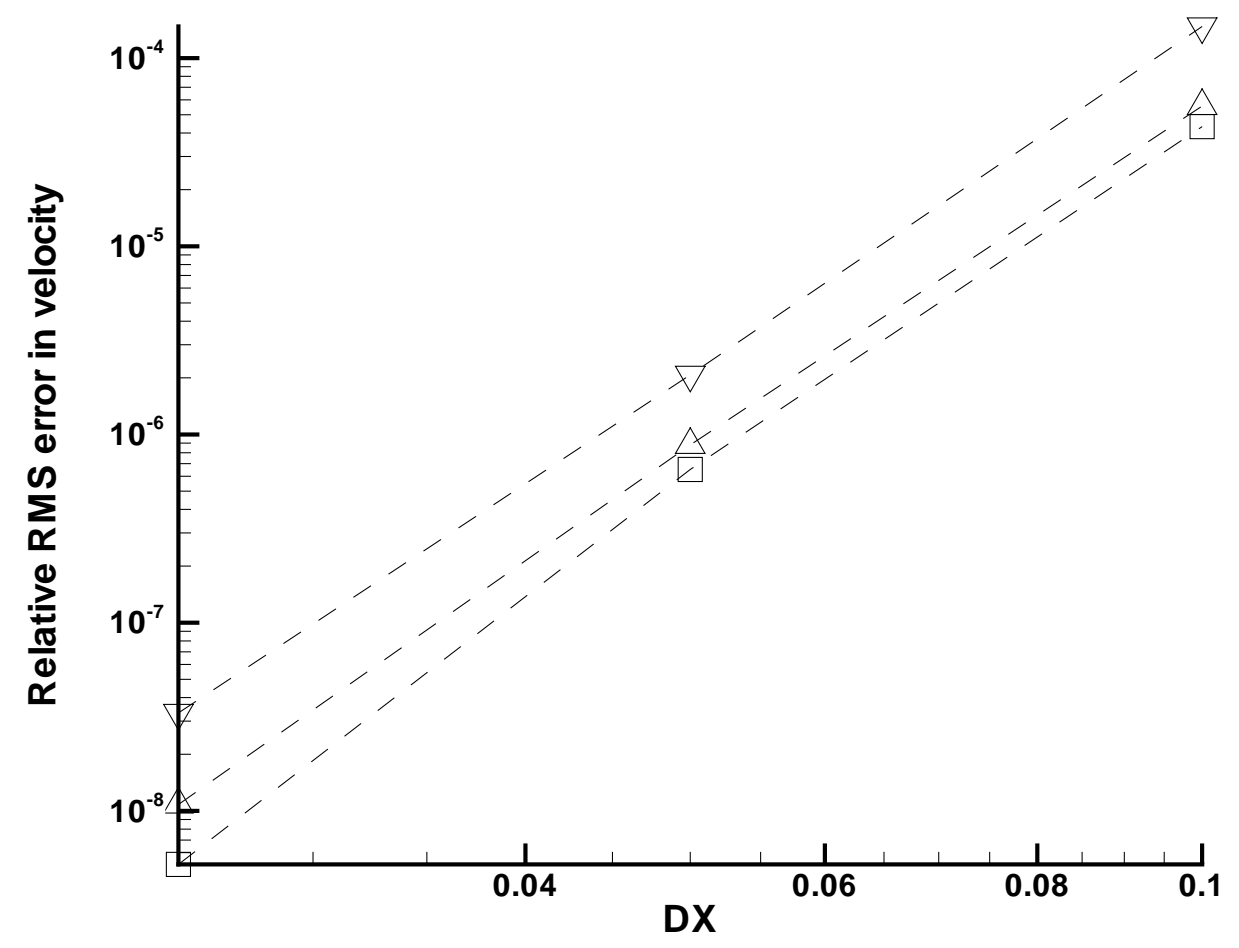

Fig. 2. RMS relative error in velocity for a convected sinusoidal density field computed with nonlinear diffusivity $\left(C_{\rho}=0.01\right)$. Squares: Mach 0.1; Delta: Mach 1; Gradients: Mac 10. 


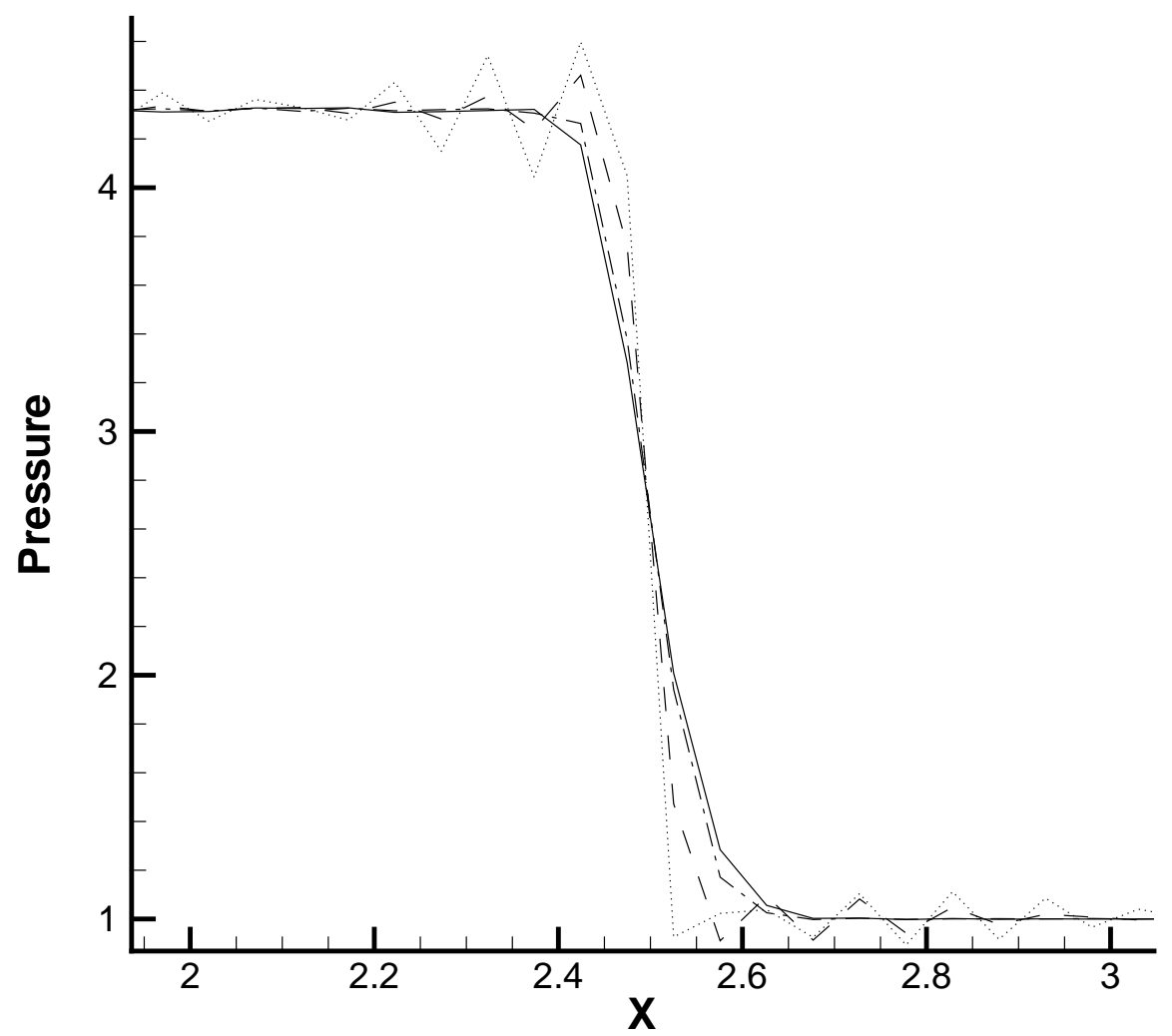

Fig. 3. The effect of model constant $C_{\mu}$ on the pressure profile across a 1-D stationary normal shock wave. Dotted line: $C_{\mu}=0$; dashed line: $C_{\mu}=0.13$; dashed-dot line: $C_{\mu}=1.0 ;$ solid line: $C_{\mu}=2.66$

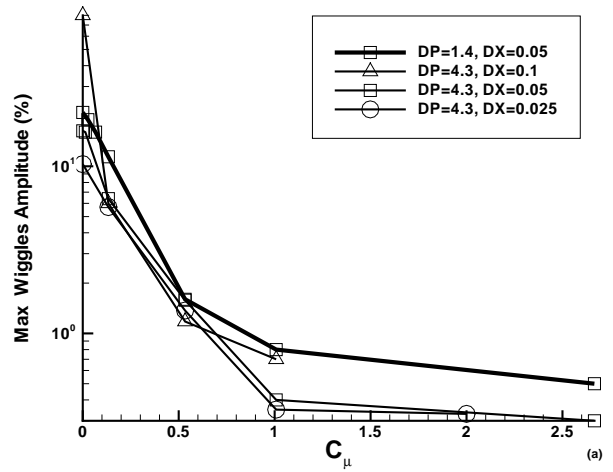

Fig. 4. Maximum normalized wiggle amplitude in $\%$ in terms of $C_{\mu}$ for the $1 \mathrm{D}$ stationary shock wave. 


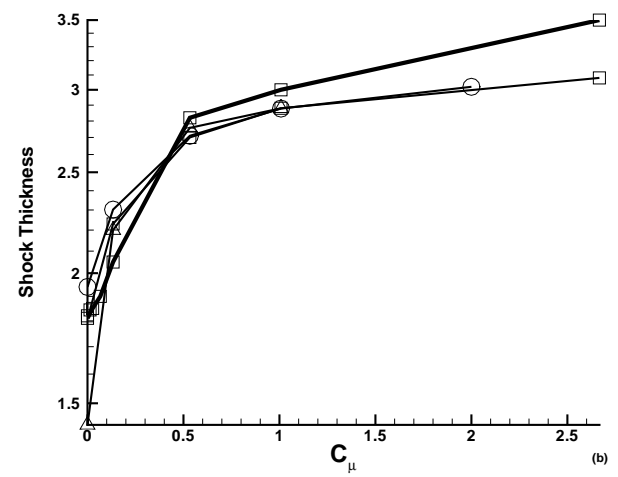

Fig. 5. Numerical shock thickness in terms of $C_{\mu}$ for the 1D stationary shock wave.

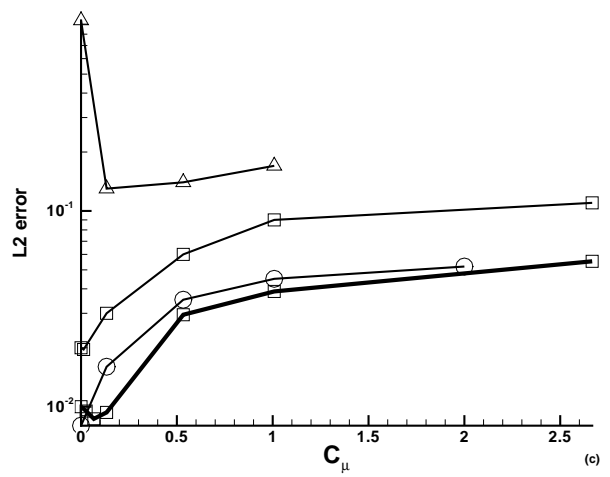

Fig. 6. L2 error in pressure in terms of $C_{\mu}$ for the 1D stationary shock wave.

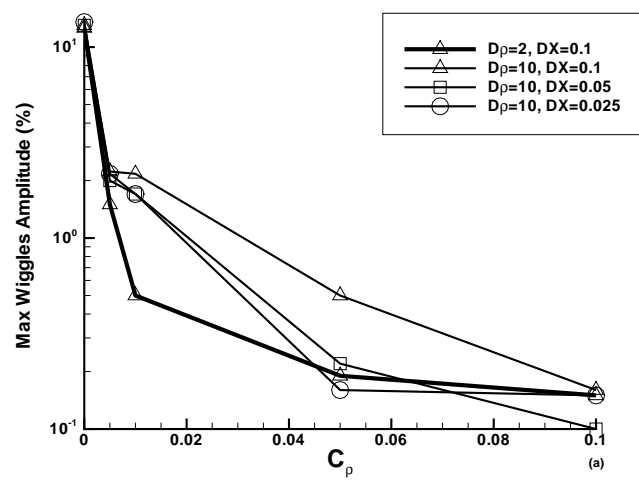

Fig. 7. Maximum normalized wiggle amplitude in $\%$ in terms of $C_{\rho}$ for the $1 \mathrm{D}$ stationary contact surface. 


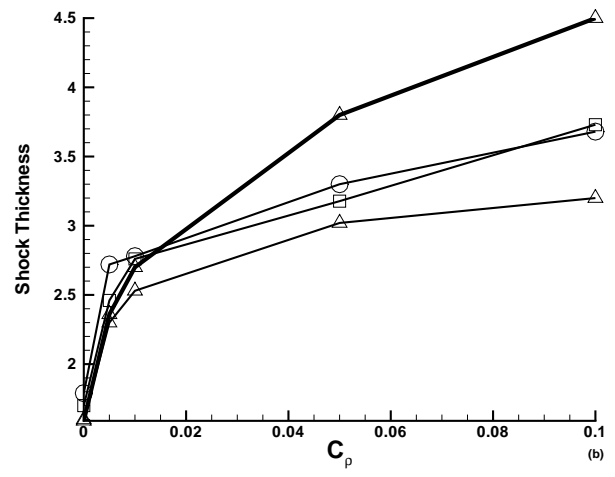

Fig. 8. Numerical shock thickness in terms of $C_{\rho}$ for the $1 \mathrm{D}$ stationary contact surface.

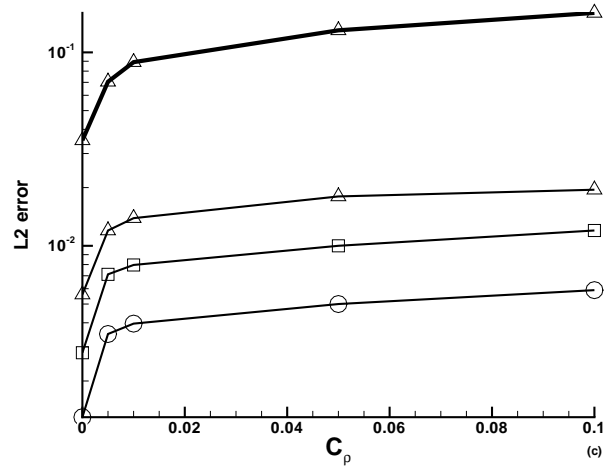

Fig. 9. L2 error in density in terms of $C_{\rho}$ for the 1D stationary contact surface. 


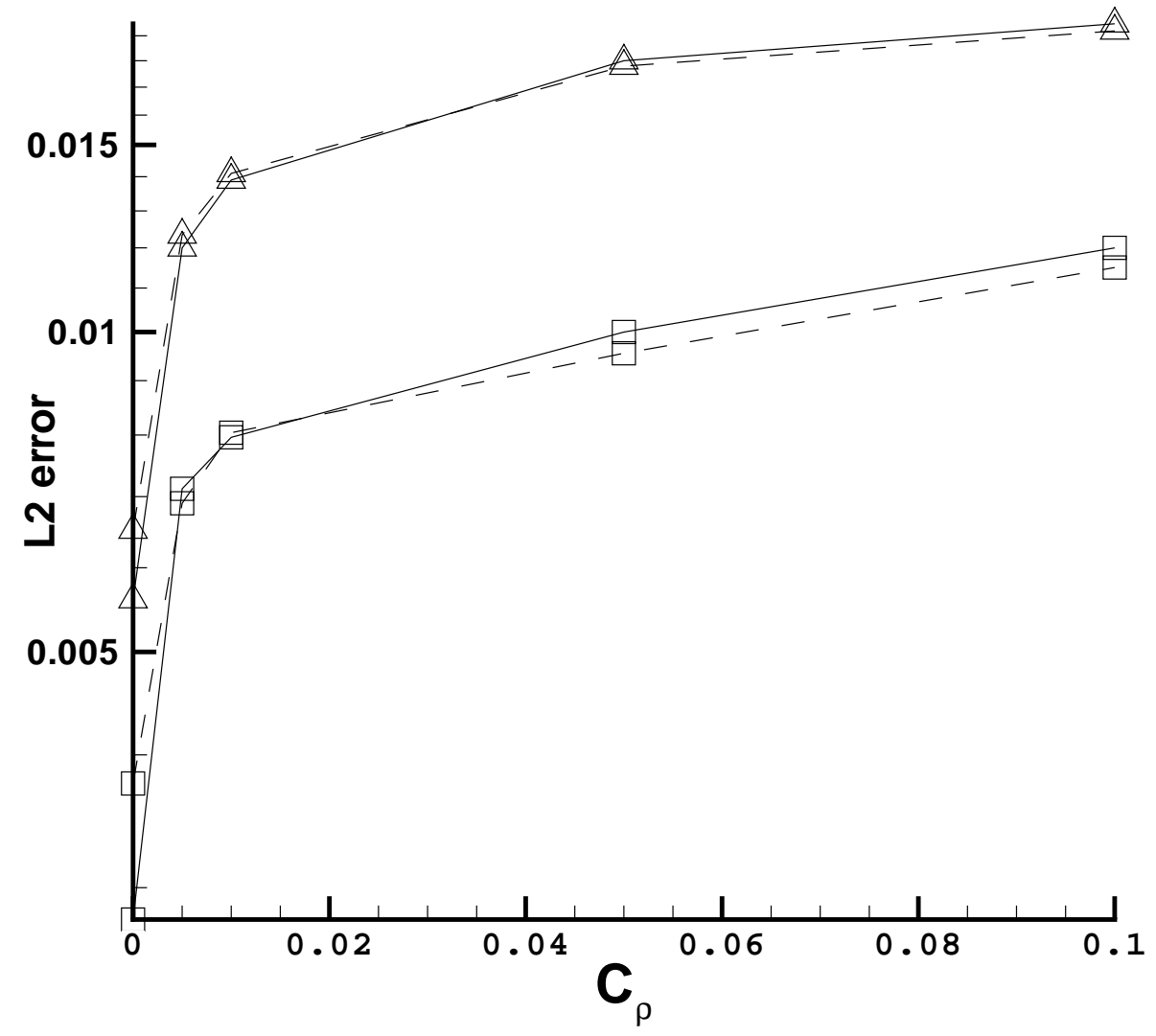

Fig. 10. L2 error in density in terms of $C_{\rho}$ for the $1 \mathrm{D}$ stationary contact surface. Solid line: Directly computed L2 error from the density field. Dashed lines: L2 error computed with the density estimated from Eq. (15); Squares: DX=0.05; Triangles: $\mathrm{DX}=0.1$ 

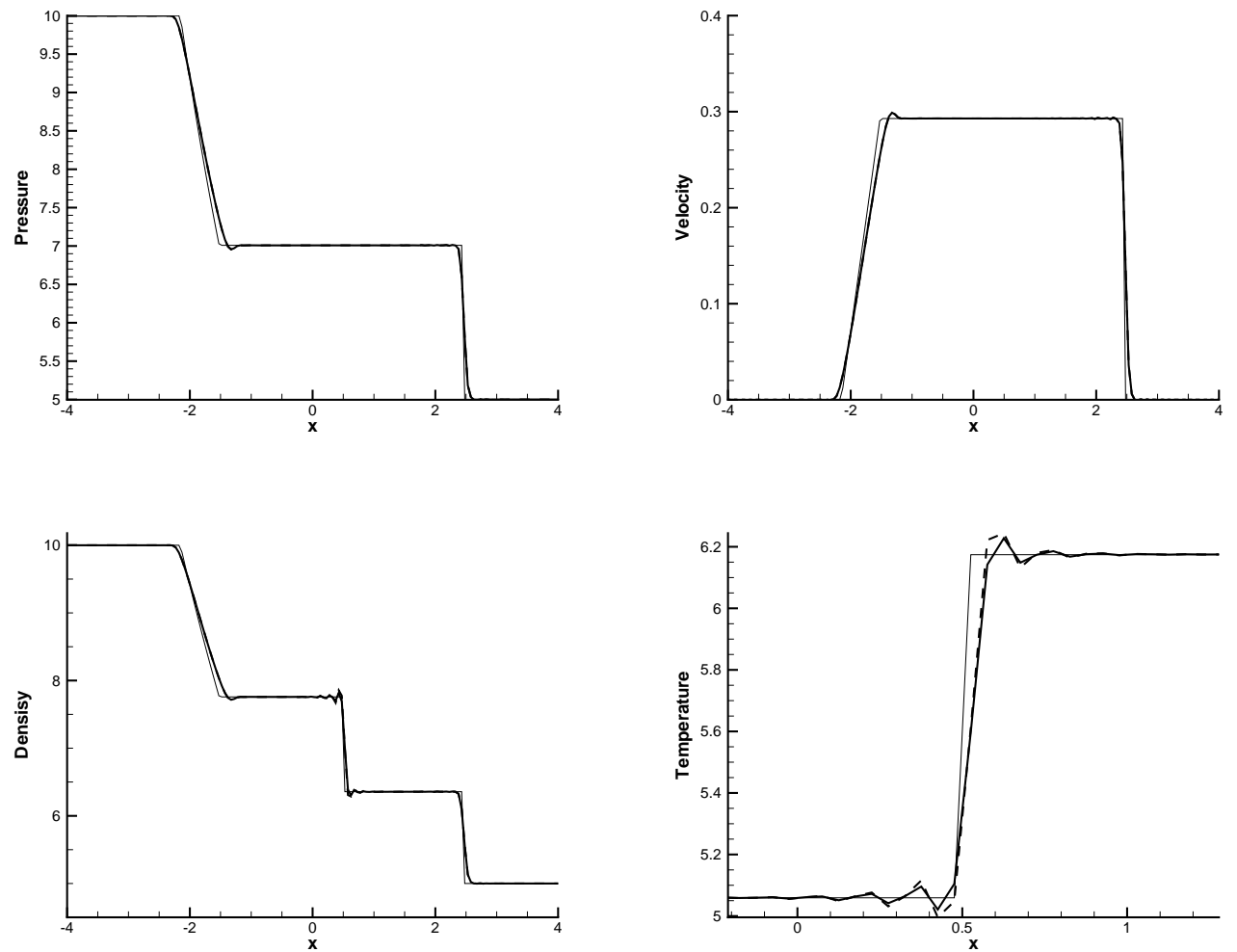

Fig. 11. Numerical simulations of the shock tube problem with an initial pressure ratio of 2. Pressure, density, velocity and temperature (zoomed in the contact discontinuity region) are expressed in terms of the physical space $X$. Thin solid line: theory; Thick dashed line: NVI model; Thick solid line: NVIDI model. 

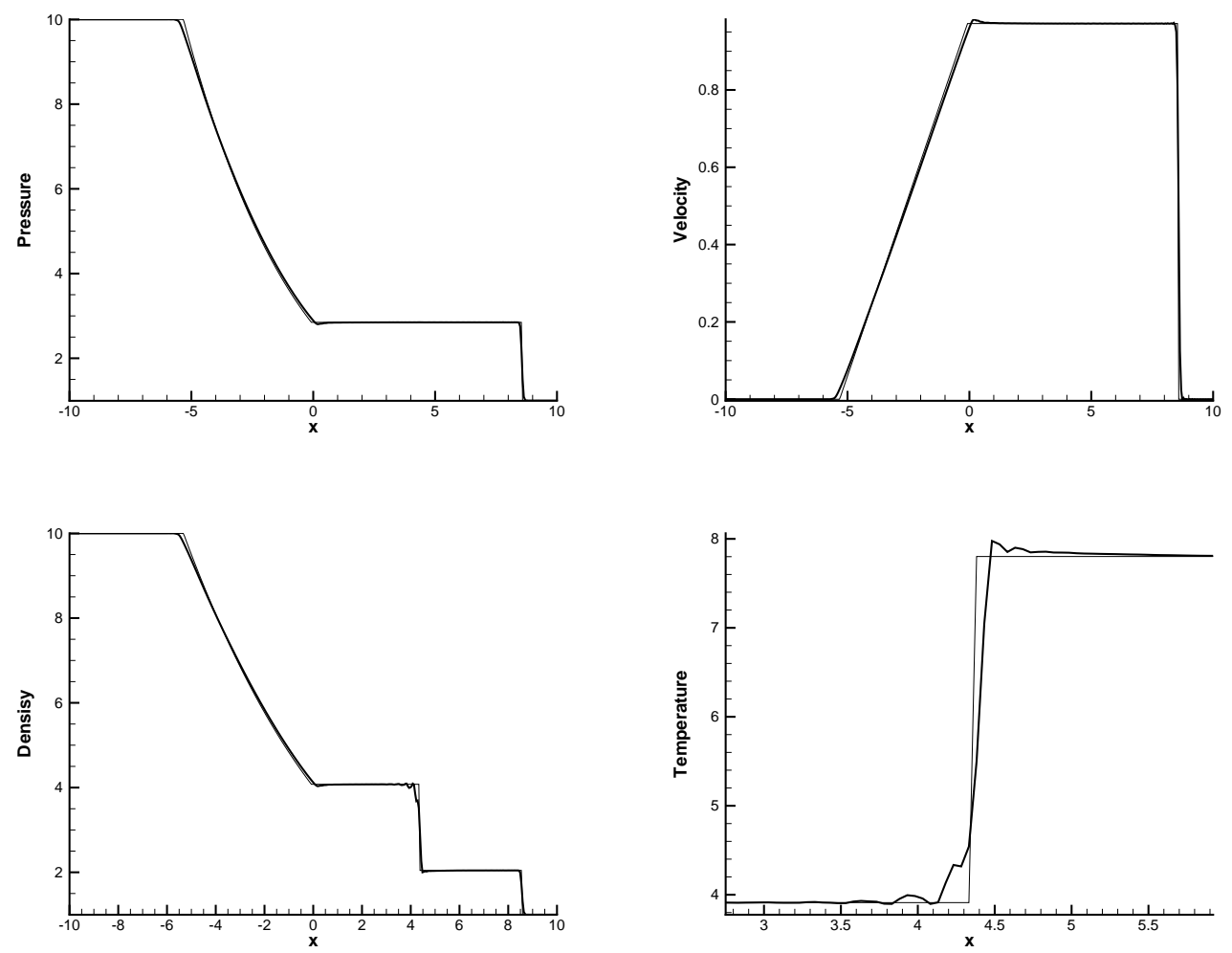

Fig. 12. Numerical simulations of the shock tube problem with an initial pressure ratio of 10. Pressure, density, velocity and temperature (zoomed in the contact discontinuity region) are expressed in terms of the physical space $X$. Thin solid line: theory; Thick solid line: NVIDI model. 

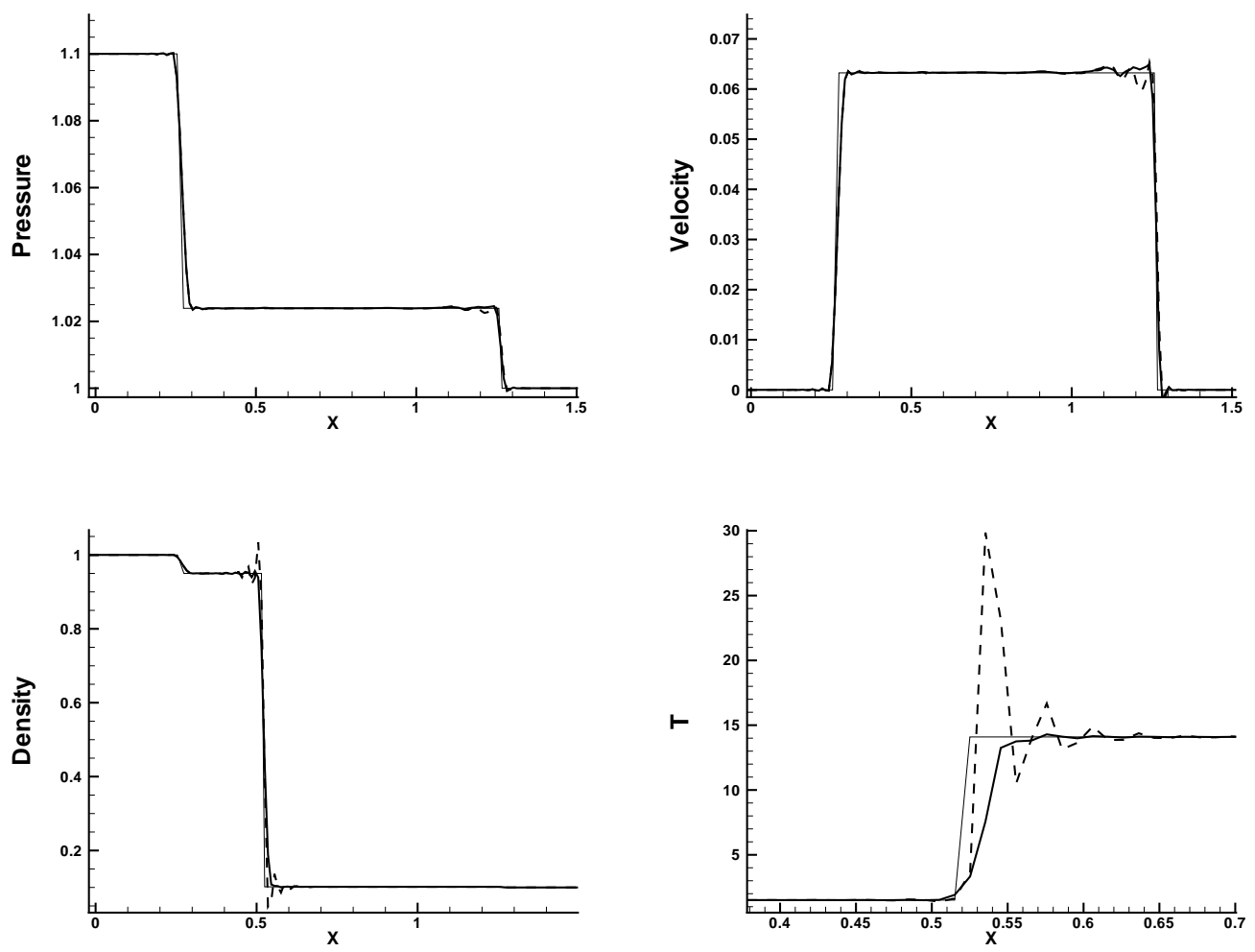

Fig. 13. Numerical simulations of the shock tube problem with a density factor of 10. Pressure, density, velocity and temperature (zoomed in the contact discontinuity region) are expressed in terms of the physical space $X$. Thin solid line: theory; Thick dashed line: NVI model; Thick solid line: NVIDI model. 


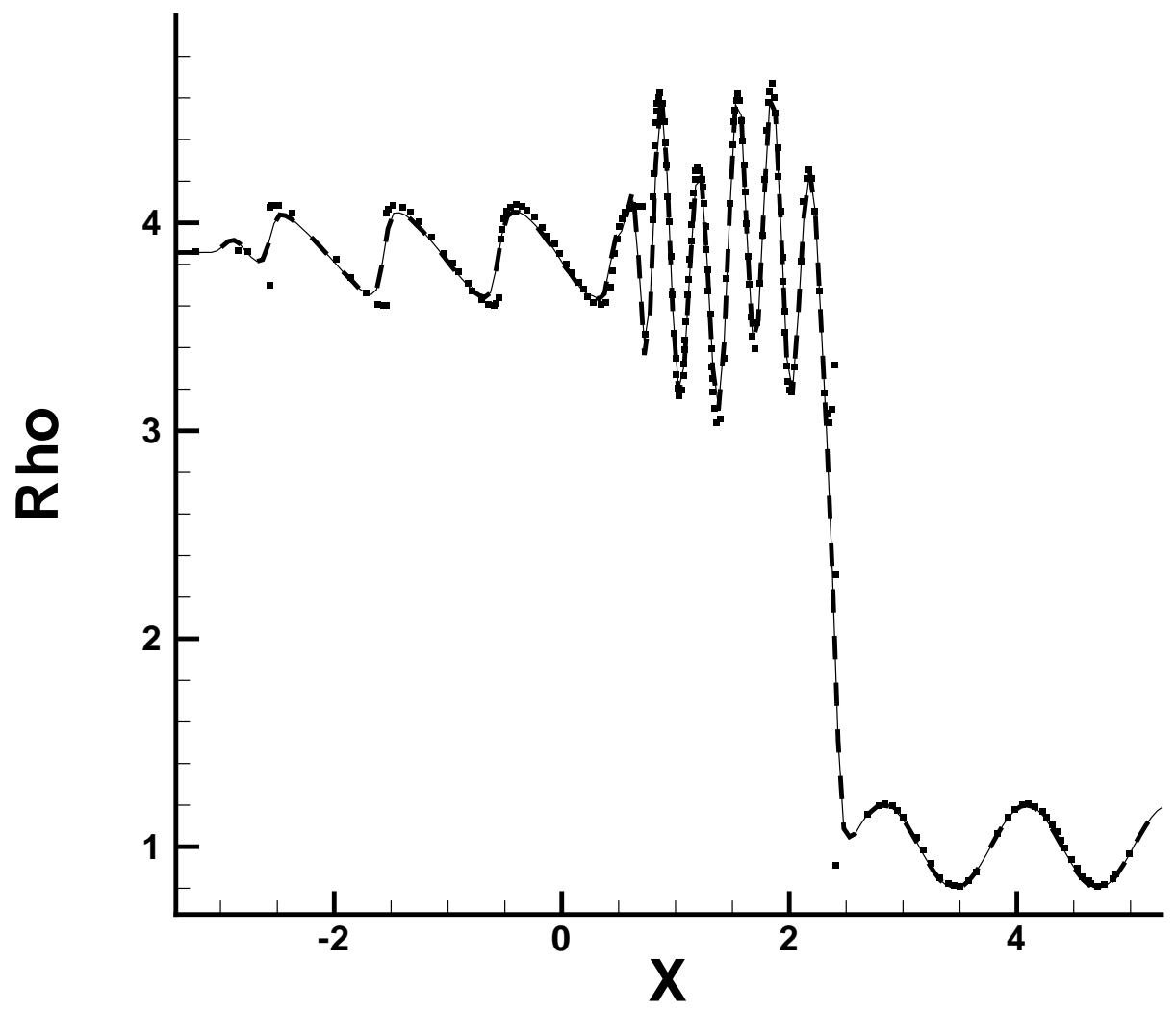

Fig. 14. A normal Mach 3 shock with pressure ratio of 10.33 is propagating into a medium with non-uniform density/entropy. Solid and dashed lines are respectively the NVI and NVIDI density solutions obtained at a resolution of 200 grid points whereas symbols represent a reference solution [14] obtained on 1600 grid points with a fifth order ENO-Roe scheme. 

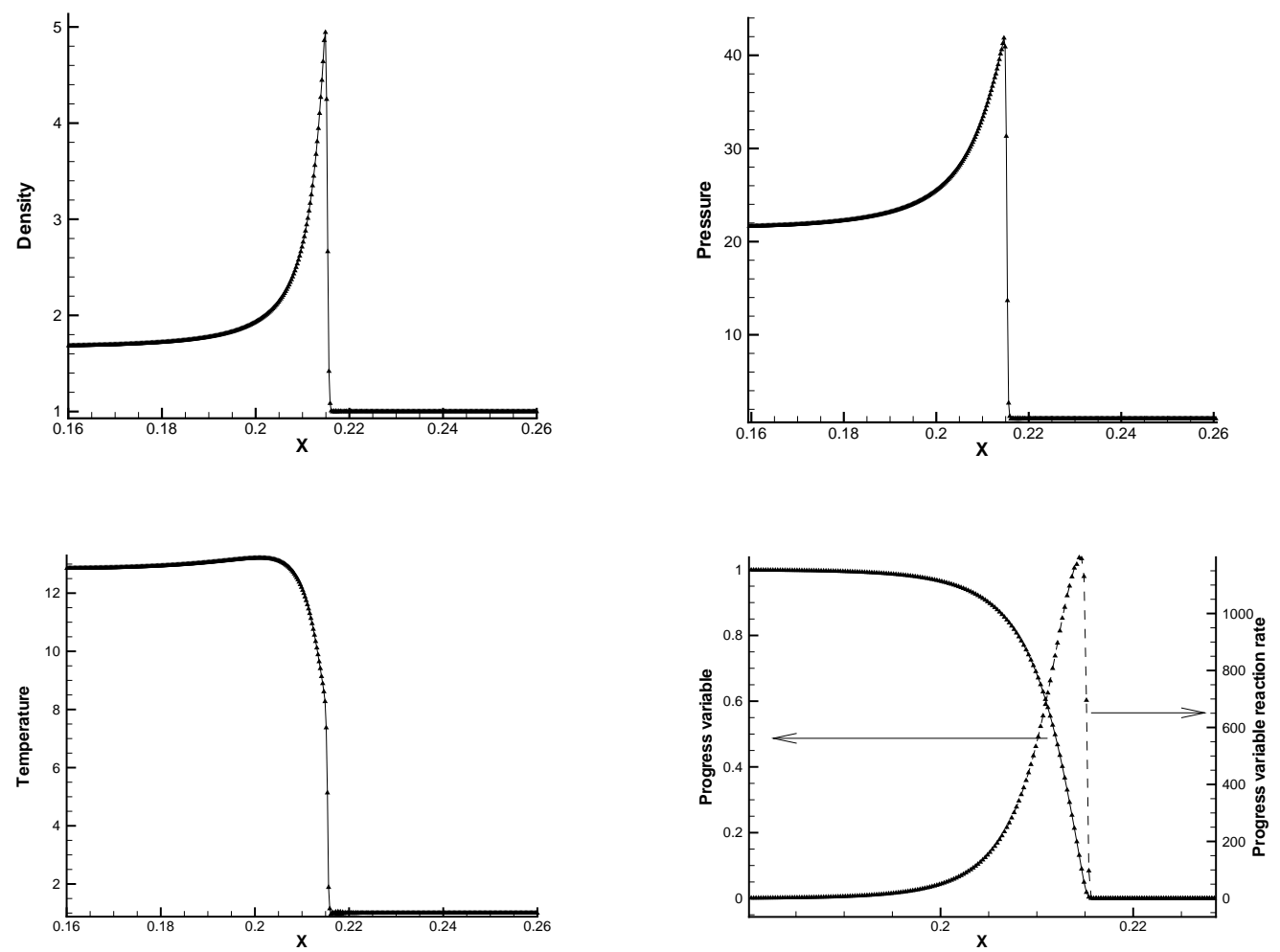

Fig. 15. Pressure, density, temperature, progress variable and reaction rate distribution for the Chapman-Jouguet detonation. 


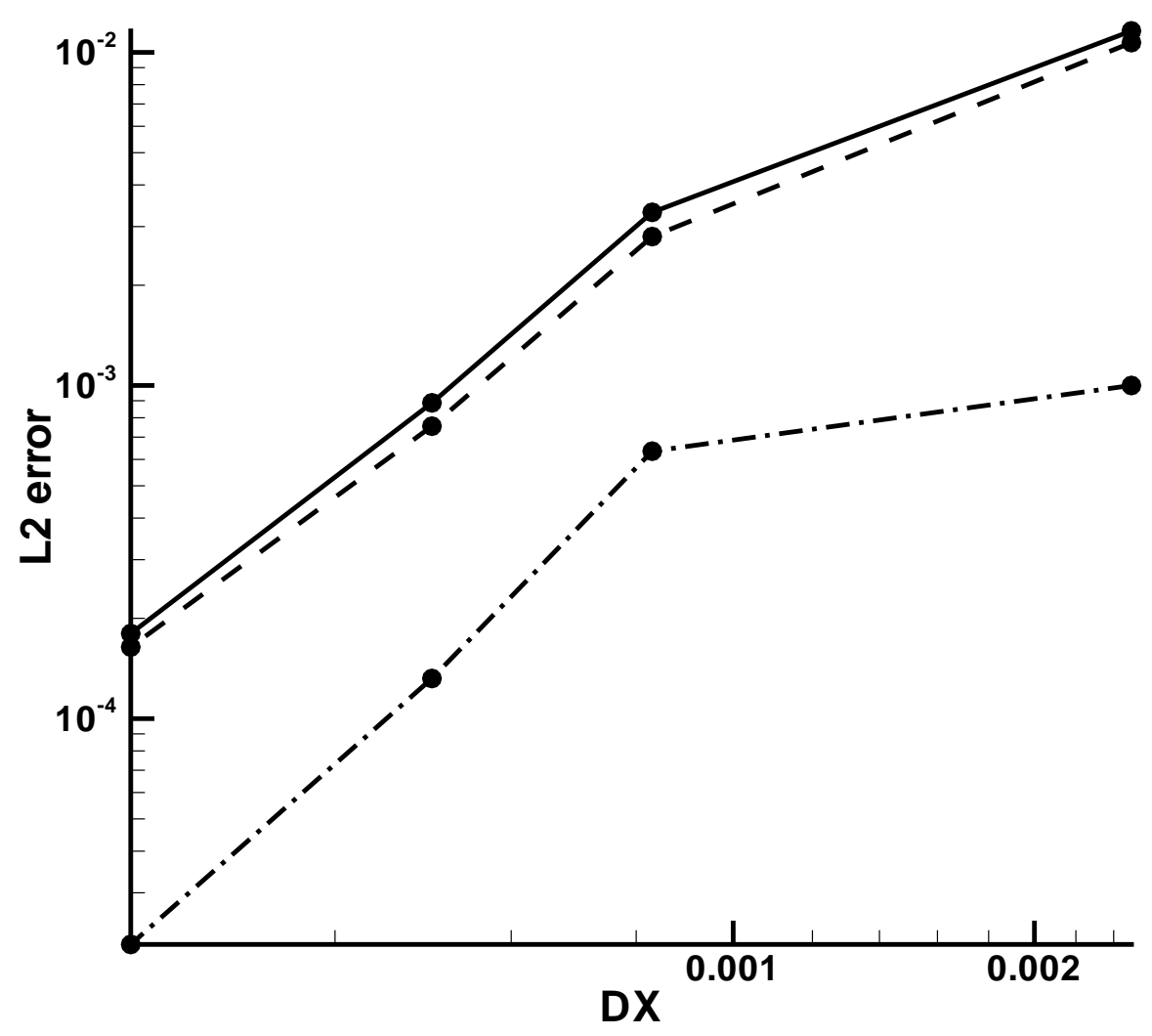

Fig. 16. L2 error in density of the CJ case in terms of the mesh size. Line: total L2 error; Dashed line: contribution of the L2 error in the reaction zone; Dashed dotted line: Contribution of the L2 error in the shock region.

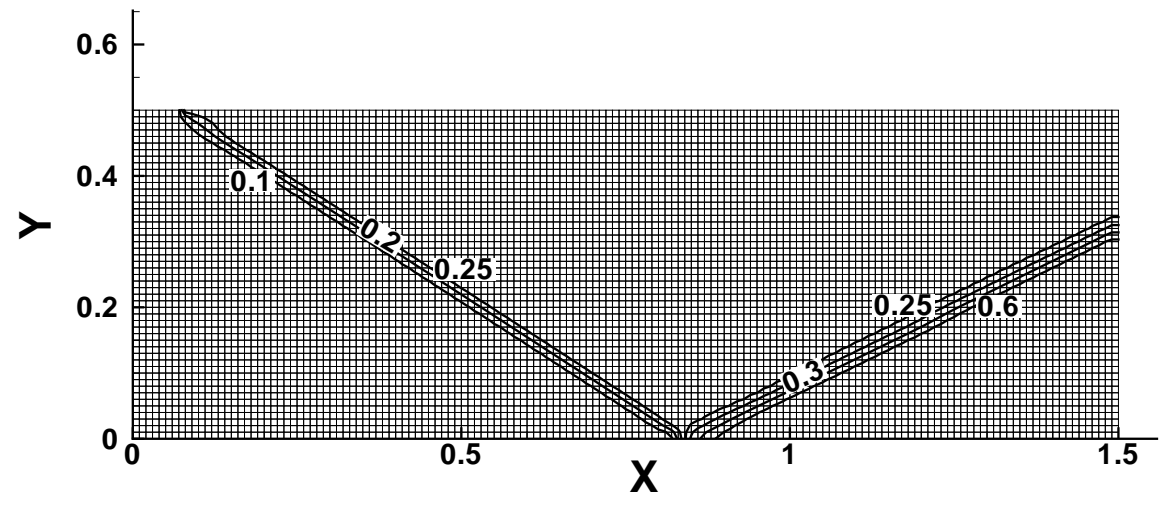

Fig. 17. The interaction of an oblique shock with an inviscid wall. The shock angle is 33 degrees from the Mach 3 free-stream. Contours of normalized pressure are plotted. 


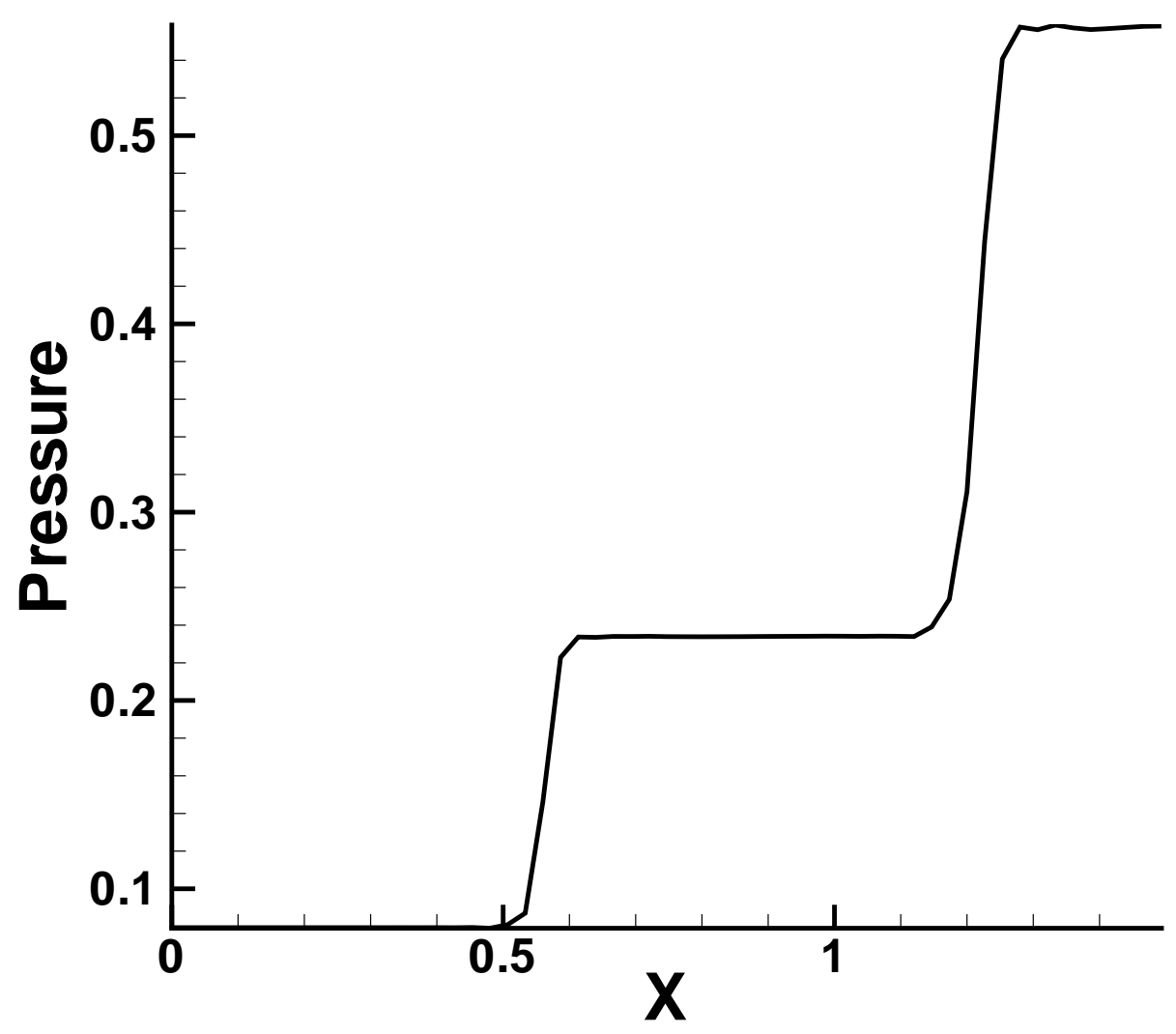

Fig. 18. Pressure distribution along $\mathrm{Y}=0.18$ line for Mach 3 inviscid shock reflection. 


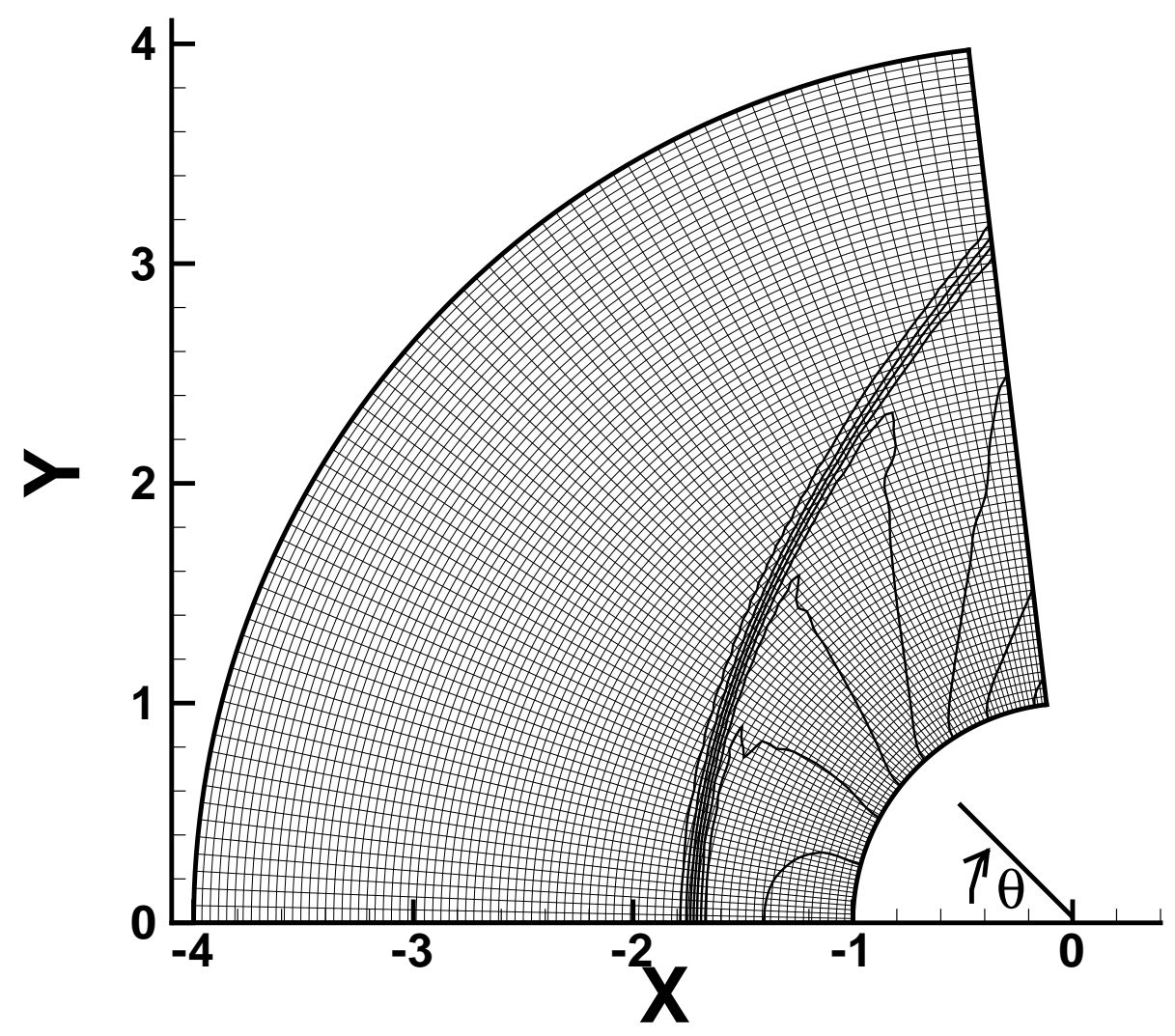

Fig. 19. Mach 3 supersonic flow past a cylinder. Countours of normalized pressure are plotted. 


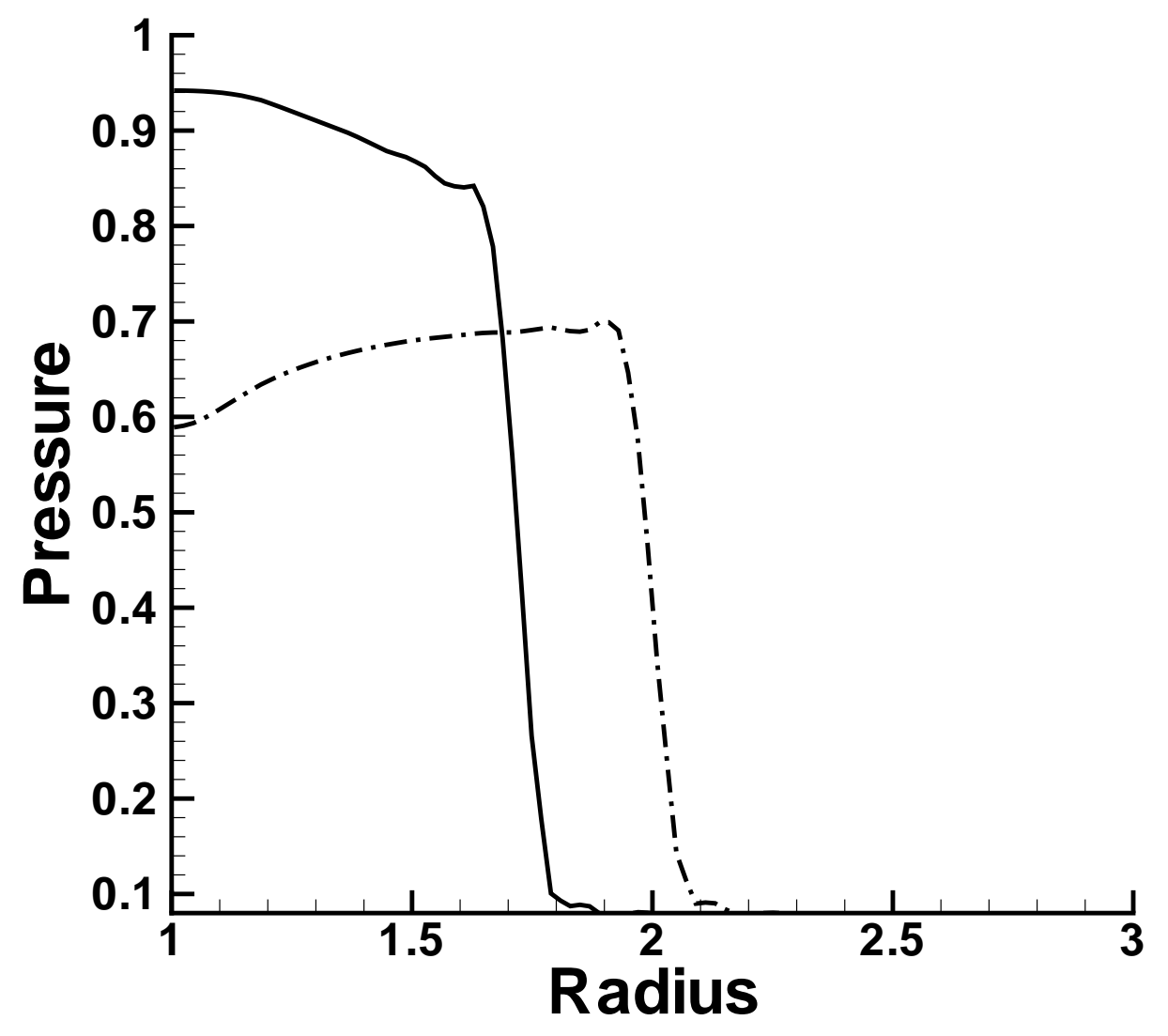

Fig. 20. Radial profiles of pressure. Solid line: $\theta=0^{\circ}$; Dashed-dotted line: $\theta=45^{\circ}$. 


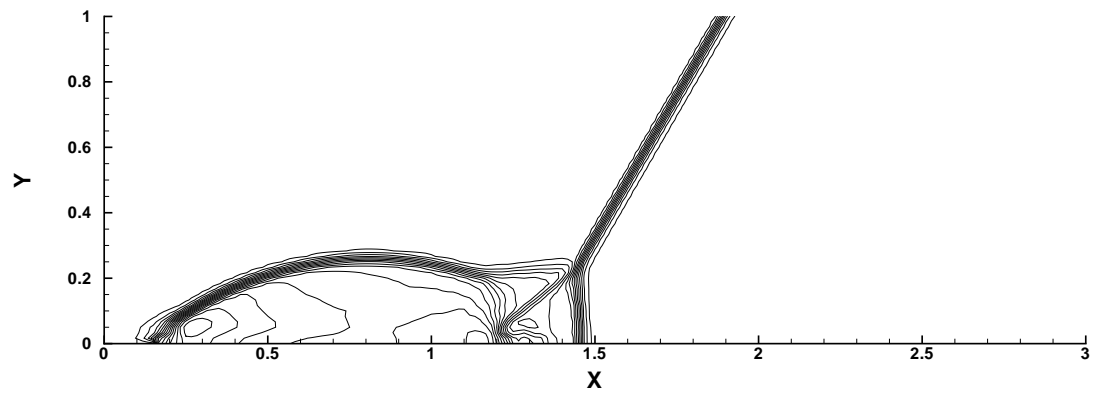

(a) $\tau=0.1$

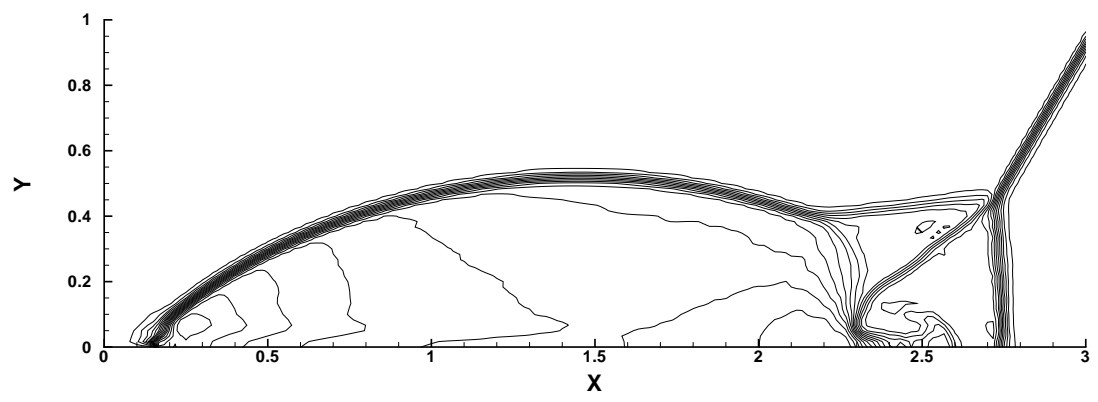

(b) $\tau=0.2$

Fig. 21. Double Mach reflection. Density, 30 contours from 1.73 to 21. 